ESAIM: COCV 27 (2021) 13

https://doi.org/10.1051/cocv/2021010
ESAIM: Control, Optimisation and Calculus of Variations

www.esaim-cocv.org

\title{
OPTIMIZATION OF NON-CYLINDRICAL DOMAINS FOR THE EXACT NULL CONTROLLABILITY OF THE 1D WAVE EQUATION
}

\author{
Arthur Bottois*, Nicolae Cîndea and Arnaud Münch
}

\begin{abstract}
This work is concerned with the null controllability of the one-dimensional wave equation over non-cylindrical distributed domains. The controllability in that case has been obtained by Castro et al. [SIAM J. Control Optim. 52 (2014)] for domains satisfying the usual geometric optic condition. We analyze the problem of optimizing the non-cylindrical support $q$ of the control of minimal $L^{2}(q)$ norm. In this respect, we prove a uniform observability inequality for a class of domains $q$ satisfying the geometric optic condition. The proof based on the d'Alembert formula relies on arguments from graph theory. Numerical experiments are discussed and highlight the influence of the initial condition on the optimal domains.
\end{abstract}

Mathematics Subject Classification. 49Q10, 93C20.

Received July 16, 2020. Accepted January 19, 2021.

\section{INTRODUCTION AND MAIN RESULTS}

In the last decades, an important literature was devoted to the study of vibrating structures controlled or stabilized using piezoelectric actuators $[1,12,27,28]$. In most of the situations, the actuators occupy a fixed position in space. Nevertheless, some studies consider the controllability of elastic structures (plates or beams) by the action of mobile piezoelectric actuators [33] which might be available in the future. Actually, a mobile actuator can be emulated by an array of fixed actuators distributed along the structure under the constraint that only one actuator can be activated at a given time [20]. The present work aims to find the optimal trajectory of a piezoelectric actuator in order to control, with a minimal cost, the vibrations of a simplified one-dimensional structure corresponding to a given initial datum. Therefore, observing the state of the considered system, we can provide in real time a trajectory for the controller in order to minimize the cost of control. More precisely, we consider the null controllability of a one-dimensional wave equation by means of distributed controls acting in non-cylindrical domains. The main contribution of this work is the proof of a uniform observability inequality with respect to control domains in a precise class. A direct consequence of this uniform observability inequality is the existence of an optimal trajectory for a piezoelectric actuator of a given size in order to control to zero the vibrations of the considered structure with a control of minimal $L^{2}$-norm.

Keywords and phrases: Wave equation, Uniform observability, Optimal shape.

Université Clermont Auvergne, Laboratoire de Mathématiques Blaise Pascal CNRS-UMR 6620, Campus des Cézeaux, 63178 Aubière cedex, France.

* Corresponding author: Arnaud.Munch@uca.fr 
Let $T$ be a positive real, $\Omega=(0,1), Q_{T}$ the domain $\Omega \times(0, T), q$ a non-empty subset of $Q_{T}$ and $\Sigma_{T}=$ $\partial \Omega \times(0, T)$. We are concerned with the null distributed controllability of the $1 \mathrm{D}$ wave equation:

$$
\begin{cases}y_{t t}-y_{x x}=v \mathbb{1}_{q} & \text { in } Q_{T} \\ y=0 & \text { on } \Sigma_{T} \\ \left(y, y_{t}\right)(\cdot, 0)=\left(y_{0}, y_{1}\right) & \text { in } \Omega .\end{cases}
$$

where $y$ represents the transverse deflection of a simplified elastic structure of length 1 and $v$ is the voltage applied to an actuator occupying the position $q_{t}=q \cap\{(x, t) ; x \in \Omega\}$ at time $t$.

We assume that $\left(y_{0}, y_{1}\right) \in \mathbf{V}:=H_{0}^{1}(\Omega) \times L^{2}(\Omega) ; v$ is the control (a function in $L^{2}(q)$ ) and $y=y(x, t)$ is the associated state. $\mathbb{1}_{q}$ from $Q_{T}$ to $\{0,1\}$ denotes the indicator function of $q$. We also use the notation

$$
L y:=y_{t t}-y_{x x}
$$

For any $\left(y_{0}, y_{1}\right) \in \mathbf{V}$ and any $v \in L^{2}(q)$, there exists exactly one solution $y$ to (1.1), with the following regularity $y \in C\left([0, T] ; H_{0}^{1}(\Omega)\right) \cap C^{1}\left([0, T] ; L^{2}(\Omega)\right)$ (see [18]). The null controllability problem for (1.1) at time $T$ is the following: for each $\left(y_{0}, y_{1}\right) \in \mathbf{V}$, find a control $v \in L^{2}(q)$ such that the corresponding solution $y$ of (1.1) satisfies

$$
\left(y, y_{t}\right)(\cdot, T)=(0,0) \text { in } \Omega
$$

As a consequence of the Hilbert uniqueness method introduced by J.-L. Lions [18], the null controllability of (1.1) is equivalent to an observability inequality for the associated adjoint problem: there exists a constant $C_{\text {obs }}(q)>0$ such that

$$
\left\|\left(\varphi_{0}, \varphi_{1}\right)\right\|_{\mathbf{W}}^{2} \leq C_{\mathrm{obs}}(q)\|\varphi\|_{L^{2}(q)}^{2}, \quad \forall\left(\varphi_{0}, \varphi_{1}\right) \in \mathbf{W}:=L^{2}(\Omega) \times H^{-1}(\Omega),
$$

where $\left(\varphi, \varphi_{0}, \varphi_{1}\right)$ solves

$$
L \varphi=0 \text { in } Q_{T}, \quad \varphi=0 \text { on } \Sigma_{T}, \quad\left(\varphi, \varphi_{t}\right)(\cdot, 0)=\left(\varphi_{0}, \varphi_{1}\right) \text { in } \Omega .
$$

$C_{\text {obs }}(q)$ is the observability constant associated with the adjoint solution $\varphi$ and depends on the control region $q$.

We address in this work the following extremal problem:

$$
\inf _{q \in \mathcal{Q}_{\mathrm{ad}}}\|v\|_{L^{2}(q)}
$$

where $v$ is the control of minimal $L^{2}(q)$-norm. The set $\mathcal{Q}_{\text {ad }}$ is defined below and denotes an admissible class of non-cylindrical domains $q$, that is domains which evolve with respect to the time variable.

In the cylindrical case for which the control region $q$ takes the form $q:=\omega \times(0, T)$, where $\omega$ denotes a subset of $\Omega$, the null controllability of (1.1) at any large $T>T^{\star}$ is well-known (for instance, see [2, 18]). The critical time $T^{\star}$ is related to the measure of the set $\Omega \backslash \bar{\omega}$. The controllability is also true in the multi-dimensional case assuming the celebrated geometric optic condition on the triplet $(\Omega, \omega, T)$ introduced by Bardos, Lebeau and Rauch in [2]. The optimal problem (1.4) has been studied by Periago in [29] with $\mathcal{Q}_{\text {ad }}=\mathcal{Q}_{\text {ad, } \tau}:=\{q=$ $\omega \times(0, T) ; \omega \subset \Omega,|\omega|=\tau|\Omega|, T \geq 2\}$ parameterized by $\tau$ in $(0,1)$. In particular, the analysis is based on the following uniform property proved by using Fourier series: there exists a constant $C>0$ which depends only on $Q_{T}$ and $\tau$ such that

$$
\sup _{q \in \mathcal{Q}_{\text {ad }, \tau}} C_{\text {obs }}(q)<C
$$


We also mention that the problem of the optimal shape and position of the support has been numerically investigated in $[23,24]$ for the one and two dimensional wave equation. This problem is also considered by Privat, Trélat and Zuazua in [30] using spectral arguments. We also mention [31] which analyze the dependence of the observability constant with respect to the control region.

The non-cylindrical case is more involved on a mathematical viewpoint. It concerns however an increasing number of studies as it allows in many situations to obtain positive controllability results, in contrast to the cylindrical case. Focusing on the wave equation, one of the first contributions is due to Khapalov [16] providing observability results for a moving point sensor in the interior of the domain. This time-dependent observation allows to avoid the usual difficulties related to strategic or non-strategic points. In particular, in the 1D setting, for any $T>0$, the existence of controls continuous almost everywhere in $(0, T)$ and supported over curves continuous almost everywhere is obtained for data in $H^{2}(\Omega) \cap H_{0}^{1}(\Omega) \times H_{0}^{1}(\Omega)$. More recently, for initial data in $\mathbf{W}$, Castro analyzes in [5] the controllability from a moving interior point. By the way of the d'Alembert formula, a uniform observability inequality is proved for a precise set of curves $\{(\gamma(t), t)\}_{t \in[0, T]}$ leading to moving controls in $H^{-1}\left(\cup_{t \in(0, T)} \gamma(t) \times t\right)$. Still in the 1D setting, Ciu, Liu and Gao [11] and Haak and Hoang [13] analyze the boundary and moving interior point observability for wave equations posed on a sufficiently regular time-dependent domain. In the $N$-dimensional case, Liu and Yong [19] employ the multiplier method to prove that the wave equation is controllable under the hypothesis that the distributed control region $q$ covers the whole space domain $\Omega$ before the time $T$. Under a similar hypothesis, we also mention the work [21] where the controllability of the damped wave equation $y_{t t}-y_{x x}-\varepsilon y_{t x x}=0$ defined on the 1D torus is established. Because of the presence of an essential spectrum, such property does not hold true in the cylindrical case.

In the 1D setting, the geometric assumption has been relaxed in [6]. Precisely, the observability inequality (1.2) is obtained assuming that the distributed control domain $q \subset Q_{T}$ is a finite union of connected open sets and satisfies the following hypothesis: any characteristic line starting from a point of $\bar{\Omega} \times\{0\}$ and following the laws of geometric optics when reflected on the boundary $\Sigma_{T}$ must meet the domain $q$ (we refer to [6], Prop. 2.1). This geometric condition is the natural extension to non-cylindrical domains of the condition introduced in [2]. Following [5], the proof of this result is obtained by the way of the d'Alembert formula. It has been extended in $[17]$ to the multi-dimensional case using microlocal analysis. We also mention the obtention of Carleman-type inequalities for general hyperbolic equations in [32].

With the aim to extend [29] to a non-cylindrical setting, we prove in a first part that the observability constant $C_{\mathrm{obs}}(q)$ in (1.2) is uniformly bounded from above for a class of domains satisfying the geometric optic condition. More precisely, for any parameter $\varepsilon>0$ small enough, we introduce the admissible set of control domains by

$$
\mathcal{Q}_{\mathrm{ad}}^{\varepsilon}=\left\{q \subset Q_{T} ; \quad q \text { open and } q^{\varepsilon} \text { verifies the geometric optic condition }\right\} .
$$

Here, $q^{\varepsilon}=q^{\varepsilon}(q)$ denotes the $\varepsilon$-interior of any $q \subset Q_{T}$ and is defined by

$$
q^{\varepsilon}=\{(x, t) \in q ; \quad d((x, t), \partial q)>\varepsilon\} .
$$

We obtain that, for any $\varepsilon>0$, there exists a constant $C>0$ which depends only on $Q_{T}$ and $\varepsilon$ such that

$$
\sup _{q \in \mathcal{Q}_{\mathrm{ad}}^{\varepsilon}} C_{\mathrm{obs}}(q)<C
$$

As in [29], this uniform property allows, in a second part, to analyze the problem (1.4) of the optimal distribution of the control domain $q$. Preliminarily, for numerical purposes, we restrict the analysis to control domains of the form

$$
q_{\gamma}=\left\{(x, t) \in Q_{T} ; \quad|x-\gamma(t)|<\delta_{0}\right\}
$$


for a given $\delta_{0}>0$ and curves $\gamma:(0, T) \rightarrow \Omega$ in the following set

$$
\mathcal{G}_{\text {ad }}=\left\{\gamma \in W^{1, \infty}(0, T) ; \quad\left\|\gamma^{\prime}\right\|_{L^{\infty}(0, T)} \leq M, \quad \delta_{0} \leq \gamma \leq 1-\delta_{0}\right\}
$$

consisting of uniformly Lipschitz functions of fixed constant $M>0$. For $T \geq 2$ and $\varepsilon>0$ small enough, the class $\left\{q_{\gamma} ; \gamma \in \mathcal{G}_{\text {ad }}\right\}$ is a subset of $\mathcal{Q}_{\text {ad }}^{\varepsilon}$. In this setting, the optimal problem (1.4) reads as follows: for $\delta_{0}>0$, $M>0$ and a given initial datum $\left(y_{0}, y_{1}\right) \in \mathbf{V}$, solve

$$
\inf _{\gamma \in \mathcal{G}_{\mathrm{ad}}}\|v\|_{L^{2}\left(q_{\gamma}\right)}^{2}
$$

where $v$ is the control of minimal $L^{2}\left(q_{\gamma}\right)$-norm distributed over $q_{\gamma} \subset Q_{T}$.

This paper is organized as follows. In Section 2, we prove the uniform observability property (1.8) on $\mathcal{Q}_{\text {ad }}^{\varepsilon}$ and its variant on the subset $\left\{q_{\gamma} ; \gamma \in \mathcal{G}_{\text {ad }}\right\}$ (see Thm. 2.1). This is achieved by defining an appropriate decomposition of the observation domains in $\mathcal{Q}_{\text {ad }}^{\varepsilon}$, and by using the d'Alembert formula. The proof also relies on arguments from graph theory. Then, in Section 3, following arguments from [15, 29], we analyze a variant of the extremal problem (1.4). Introducing a $C^{1}$-regularization of the support $q_{\gamma}$, we prove that the underlying cost is continuous over $\mathcal{G}_{\text {ad }}$ for the $L^{\infty}(0, T)$-norm, and admits at least one local minimum (see Prop. 2). Section 4 illustrates the result with numerical experiments for the regularized minimization problem introduced in Section 3.3. Minimization sequences for the regularized cost are constructed using a gradient method: each iteration requires the computation of a null control, performed using the space-time formulation developed in [9] and used in [6], well-suited to the description of the non-cylindrical domains where the control acts. As expected, the optimal domain we obtain are closely related to the travelling waves generated by the initial condition. Section 5 concludes the work with some perspectives.

\section{UNIFORM OBSERVABILITY WITH RESPECT TO THE DOMAIN OF OBSERVATION}

We prove in this section the uniform observability property (1.8) with respect to the domain of observation. Precisely, we prove the following equivalent result for regular data in $\mathbf{V}$.

Theorem 2.1. Let $T>0$ and let $\varepsilon>0$ be a small enough fixed parameter such that the set $\mathcal{Q}_{\text {ad }}^{\varepsilon}$ defined by (1.6) is non-empty. There exists a constant $C_{\mathrm{obs}}^{\varepsilon}>0$ such that for every $q \in \mathcal{Q}_{\mathrm{ad}}^{\varepsilon}$, the following inequality holds

$$
\left\|\left(\varphi_{0}, \varphi_{1}\right)\right\|_{\mathbf{V}}^{2} \leq C_{\mathrm{obs}}^{\varepsilon}\left\|\varphi_{t}\right\|_{L^{2}(q)}^{2}, \quad \forall\left(\varphi_{0}, \varphi_{1}\right) \in \mathbf{V}
$$

where $\varphi$ is the solution of the wave equation (1.3) associated with the initial datum $\left(\varphi_{0}, \varphi_{1}\right)$.

We emphasize that the constant $C_{\text {obs }}^{\varepsilon}$ does not depend on $q \in \mathcal{Q}_{\text {ad }}^{\varepsilon}$. We also emphasize that the parameter $\varepsilon$ is introduced here to quantify to which extent a domain $q$ satisfies the geometric optic condition used in [6]. As $\varepsilon$ goes to zero, the observability constant $C_{\mathrm{obs}}^{\varepsilon}$ blows up (see Rem. 2.18). The same is true when $\tau$ goes to zero in the study [29], see (1.5). Eventually, we emphasize that any domain $q \in \mathcal{Q}_{\text {ad }}^{\varepsilon}$ for some $\varepsilon$ satisfies the geometric optic condition. Conversely, for any domain $q$ satisfying the geometric optic condition, there exists $\varepsilon>0$ such that $q \in \mathcal{Q}_{\mathrm{ad}}^{\varepsilon}$.

In the remaining part of this section, we assume that the hypotheses of Theorem 2.1 are satisfied.

\subsection{Proof of Theorem 2.1}

The idea of the proof is to decompose the domain $Q_{T}$ as the sum of "elementary squares" on which, in view of the d'Alembert formula, the terms in the inequality (2.1) can be simply computed and compared. The proof based on arguments from graph theory allows notably to relate the value of the observability constant to the spectrum of a Laplacian matrix, defined in terms of the graph associated to any domain $q \in \mathcal{Q}_{\text {ad }}^{\varepsilon}$. 
The proof requires some notations and technical lemmas. We decompose it into seven steps.

Step 1 - Solution of the adjoint problem (1.3) associated with piecewise affine initial conditions.

Let $N \in \mathbb{N}^{*}$ and $\kappa_{N}=1 / N$. We denote by $S_{N}=\left(x_{i}^{N}\right)_{0<i<N}$ the regular subdivision of $\bar{\Omega}$ in $N$ intervals such that $x_{i}^{N}=i / N$. For any $\left(\varphi_{0}, \varphi_{1}\right) \in \mathbf{V}$, we then associate the functions $\varphi_{0}^{N}, \varphi_{1}^{N}$ as follows: for all $x \in \Omega$, we set

$$
\begin{aligned}
& \varphi_{0}^{N}(x)=\sum_{i=1}^{N}\left(\varphi_{0}\left(x_{i}^{N}\right) \frac{x-x_{i-1}^{N}}{\kappa_{N}}+\varphi_{0}\left(x_{i-1}^{N}\right) \frac{x_{i}^{N}-x}{\kappa_{N}}\right) \mathbb{1}_{\left[x_{i-1}^{N}, x_{i}^{N}\right]}(x), \\
& \varphi_{1}^{N}(x)=\sum_{i=1}^{N} \beta_{i}^{N} \mathbb{1}_{\left[x_{i-1}^{N}, x_{i}^{N}\right]}(x), \quad \text { with } \beta_{i}^{N}=\frac{1}{\kappa_{N}} \int_{x_{i-1}^{N}}^{x_{i}^{N}} \varphi_{1},
\end{aligned}
$$

which are respectively affine and constant on each interval $\left[x_{i-1}^{N}, x_{i}^{N}\right]$. We also denote by $\left(\varphi_{0}^{N}\right)^{\prime} \in L^{2}(\Omega)$ the "derivative" of $\varphi_{0}^{N}$ :

$$
\left(\varphi_{0}^{N}\right)^{\prime}(x)=\sum_{i=1}^{N} \alpha_{i}^{N} \mathbb{1}_{\left[x_{i-1}^{N}, x_{i}^{N}\right]}(x), \quad \text { with } \alpha_{i}^{N}=\frac{\varphi_{0}\left(x_{i}^{N}\right)-\varphi_{0}\left(x_{i-1}^{N}\right)}{\kappa_{N}} .
$$

Using that $\varphi_{0} \in H_{0}^{1}(\Omega)$, we easily check that

$$
\left\|\varphi_{0}^{N}\right\|_{H_{0}^{1}(\Omega)}^{2}=\frac{1}{N} \sum_{i=1}^{N}\left(\alpha_{i}^{N}\right)^{2}, \quad\left\|\varphi_{1}^{N}\right\|_{L^{2}(\Omega)}^{2}=\frac{1}{N} \sum_{i=1}^{N}\left(\beta_{i}^{N}\right)^{2} \quad \text { and } \quad \sum_{i=1}^{N} \alpha_{i}^{N}=0 .
$$

In order to use the d'Alembert formula for the solution of (1.3) associated with the initial datum $\left(\varphi_{0}^{N}, \varphi_{1}^{N}\right)$, we extend these functions as odd functions to $[-1,1]$ and then by 2-periodicity to $\mathbb{R}$. In this respect, we first extend the definition of $x_{i}^{N}$ to $i \in \mathbb{Z}$ by putting $x_{i}^{N}=i / N$ for every $i \in \mathbb{Z}$, and then denote by $I_{i}^{N}$ for every $i \in \mathbb{Z}^{*}$ the following interval:

$$
I_{i}^{N}= \begin{cases}{\left[x_{i-1}^{N}, x_{i}^{N}\right]} & \text { if } i>0 \\ {\left[x_{i}^{N}, x_{i+1}^{N}\right]} & \text { if } i<0\end{cases}
$$

Definition 2.2. For any $N \in \mathbb{N}^{*}$ and $i \in \mathbb{Z}^{*}$, we define the integer $\mathfrak{j}_{N}(i)$ as follows:

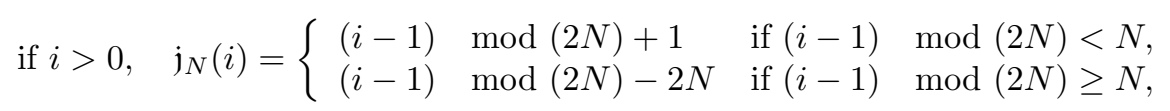

and if $i<0, \mathfrak{j}_{N}(i)=-\mathfrak{j}_{N}(-i)$. Remark that for every $i \in \mathbb{Z}^{*}, \mathfrak{j}_{N}(i) \in \mathbb{I}_{N}$ with

$$
\mathbb{I}_{N}=\{-N, \ldots,-1,1, \ldots, N\} .
$$

Similarly, we extend $\alpha_{i}^{N}$ and $\beta_{i}^{N}$ for every $i \in \mathbb{Z}^{*}$ as follows: if $i \in\{-N, \ldots,-1\}$, we set $\alpha_{i}^{N}=\alpha_{-i}^{N}$ and $\beta_{i}^{N}=-\beta_{-i}^{N}$; if $|i|>N$, we set $\alpha_{i}^{N}=\alpha_{\mathbf{j}_{N}(i)}^{N}$ and $\beta_{i}^{N}=\beta_{\mathbf{j}_{N}(i)}^{N}$.

Definition 2.3. For any $N \in \mathbb{N}^{*}$ and $i \in \mathbb{I}_{N}$, let

$$
\gamma_{i}^{N}=\alpha_{i}^{N}+\beta_{i}^{N}
$$

We extend the functions $\varphi_{0}^{N}$ and $\varphi_{1}^{N}$ as odd functions to $[-1,1]$ and by 2-periodicity to $\mathbb{R}$. 
Lemma 2.4. For any $N \in \mathbb{N}^{*}$, the solution $\varphi^{N}$ of (1.3) associated with the initial datum $\left(\varphi_{0}^{N}, \varphi_{1}^{N}\right)$ satisfies:

$$
\varphi_{t}^{N}(x, t)=\frac{1}{2} \sum_{i \in \mathbb{Z}^{*}} \sum_{j \in \mathbb{Z}^{*}}\left(\gamma_{\mathfrak{j}_{N}(i)}^{N}-\gamma_{-\mathfrak{j}_{N}(j)}^{N}\right) \mathbb{1}_{I_{i}^{N}}(x+t) \mathbb{1}_{I_{j}^{N}}(x-t), \quad \forall(x, t) \in Q_{T}
$$

Proof. Using the notations above, we obtain

$$
\left(\varphi_{0}^{N}\right)^{\prime}(x)=\sum_{i \in \mathbb{Z}^{*}} \alpha_{i}^{N} \mathbb{1}_{I_{i}^{N}}(x), \quad \varphi_{1}^{N}(x)=\sum_{i \in \mathbb{Z}^{*}} \beta_{i}^{N} \mathbb{1}_{I_{i}^{N}}(x), \quad \forall x \in \mathbb{R}
$$

Moreover, from the d'Alembert formula, the solution $\varphi^{N}$ of (1.3) associated with the initial datum $\left(\varphi_{0}^{N}, \varphi_{1}^{N}\right)$ is given as follows:

$$
\varphi^{N}(x, t)=\frac{1}{2}\left(\varphi_{0}^{N}(x+t)+\varphi_{0}^{N}(x-t)\right)+\frac{1}{2} \int_{x-t}^{x+t} \varphi_{1}^{N}, \quad \forall(x, t) \in Q_{T}
$$

Taking the derivative with respect to $t$ and replacing the expressions (2.9) in the above equation, we deduce that for all $(x, t) \in Q_{T}$, we have

$$
\begin{aligned}
\varphi_{t}^{N}(x, t) & =\frac{1}{2}\left(\left(\varphi_{0}^{N}\right)^{\prime}(x+t)-\left(\varphi_{0}^{N}\right)^{\prime}(x-t)+\varphi_{1}^{N}(x+t)+\varphi_{1}^{N}(x-t)\right) \\
& =\frac{1}{2} \sum_{i \in \mathbb{Z}^{*}}\left(\left(\alpha_{i}^{N}+\beta_{i}^{N}\right) \mathbb{1}_{I_{i}^{N}}(x+t)-\left(\alpha_{i}^{N}-\beta_{i}^{N}\right) \mathbb{1}_{I_{i}^{N}}(x-t)\right) \\
& =\frac{1}{2} \sum_{i \in \mathbb{Z}^{*}} \sum_{j \in \mathbb{Z}^{*}}\left(\alpha_{i}^{N}+\beta_{i}^{N}-\alpha_{j}^{N}+\beta_{j}^{N}\right) \mathbb{1}_{I_{i}^{N}}(x+t) \mathbb{1}_{I_{j}^{N}}(x-t) .
\end{aligned}
$$

Using the properties of the function $\mathfrak{j}_{N}$ defined in (2.5), we deduce that for $i, j \in \mathbb{Z}^{*}$,

$$
\begin{aligned}
\alpha_{i}^{N}+\beta_{i}^{N}-\alpha_{j}^{N}+\beta_{j}^{N} & =\alpha_{\mathfrak{j}_{N}(i)}^{N}+\beta_{\mathfrak{j}_{N}(i)}^{N}-\alpha_{\mathfrak{j}_{N}(j)}^{N}+\beta_{\mathfrak{j}_{N}(j)}^{N} \\
& =\left(\alpha_{\mathfrak{j}_{N}(i)}^{N}+\beta_{\mathfrak{j}_{N}(i)}^{N}\right)-\left(\alpha_{-\mathfrak{j}_{N}(j)}^{N}+\beta_{-\mathfrak{j}_{N}(j)}^{N}\right) \\
& =\gamma_{\mathfrak{j}_{N}(i)}^{N}-\gamma_{-\mathfrak{j}_{N}(j)}^{N}
\end{aligned}
$$

and the result.

Step 2 - Representation of $q \in \mathcal{Q}_{\text {ad }}^{\varepsilon}$ using "elementary squares".

In view of the expression (2.8), we introduce the following definition.

Definition 2.5. For any $N \in \mathbb{N}^{*}$ and $i, j \in \mathbb{Z}^{*}$, we define the elementary square of indices $(i, j)$ associated with the subdivision $S_{N}$ as the following closed set of $\mathbb{R}^{2}$ :

$$
C_{(i, j)}^{N}=\left\{(x, t) \in \mathbb{R}^{2} \text { such that } x+t \in I_{i}^{N} \text { and } x-t \in I_{j}^{N}\right\},
$$

where the interval $I_{i}^{N}$ is given by (2.4).

We also denote by $\mathcal{C}_{N}=\left\{C_{(i, j)}^{N} ; i, j \in \mathbb{Z}^{*}\right\}$ the set of all the elementary squares associated with the subdivision $S_{N}$. We easily check that $\mathbb{R}^{2}=\bigcup_{i, j \in \mathbb{Z}^{*}} C_{(i, j)}^{N}$.

Figure 1 illustrates the way the elementary squares are indexed, with the example of the subdivision $S_{4}$. 


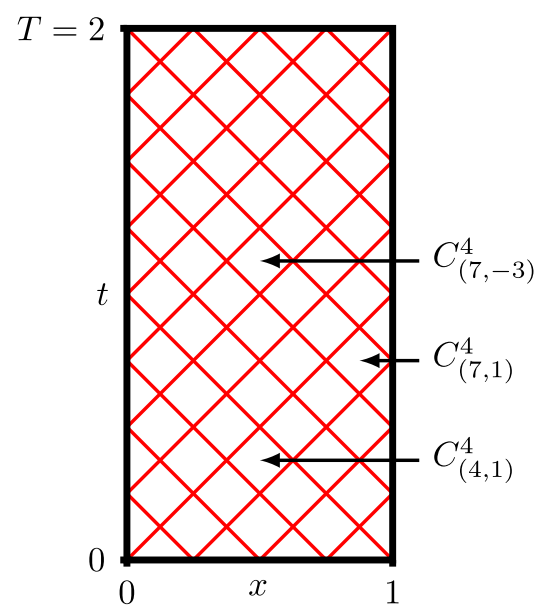

Figure 1. Some elementary squares in $\mathcal{C}_{4}$.

Remark 2.6. For every $i, j \in \mathbb{Z}^{*}$, the coordinates of the center of the elementary square $C_{(i, j)}^{N}$ associated with the subdivision $S_{N}$ are given by

$$
\left\{\begin{array}{l}
x_{(i, j)}^{N}=\frac{m_{i}^{N}+m_{j}^{N}}{2}, \\
t_{(i, j)}^{N}=\frac{m_{i}^{N}-m_{j}^{N}}{2},
\end{array} \quad \text { with } \quad m_{i}^{N}= \begin{cases}\frac{x_{i-1}^{N}+x_{i}^{N}}{2} & \text { if } i>0, \\
\frac{x_{i}^{N}+x_{i+1}^{N}}{2} & \text { if } i<0 .\end{cases}\right.
$$

The area of every elementary square $C_{(i, j)}^{N} \in \mathcal{C}_{N}$ is given by $\left|C_{(i, j)}^{N}\right|=\frac{1}{2 N^{2}}$. Notice that for every $i, j \in \mathbb{Z}^{*}$ with $|i|,|j|>1$, the elementary squares having one side in common with the elementary square $C_{(i, j)}^{N}$ are $C_{(i \pm 1, j)}^{N}$ and $C_{(i, j \pm 1)}^{N}$.

Definition 2.7. For every $q \in \mathcal{Q}_{\text {ad }}^{\varepsilon}$, we denote by $\mathcal{C}_{N}(q)$ and $\mathcal{C}_{N}\left(Q_{T}\right)$ the sets of the elementary squares in $\mathcal{C}_{N}$ with their interior included in $q$ and $Q_{T}$ respectively:

$$
\mathcal{C}_{N}(q)=\left\{C_{(i, j)}^{N} \in \mathcal{C}_{N} ; \quad \stackrel{\circ}{C}_{(i, j)}^{N} \subset q\right\}, \quad \mathcal{C}_{N}\left(Q_{T}\right)=\left\{C_{(i, j)}^{N} \in \mathcal{C}_{N} ; \quad \stackrel{\circ}{C}_{(i, j)}^{N} \subset Q_{T}\right\}
$$

If $N$ is large enough, the sets $\mathcal{C}_{N}(q)$ and $\mathcal{C}_{N}\left(Q_{T}\right)$ are non-empty.

We also define $R_{N}(q)$ as the union of the elementary squares in $\mathcal{C}_{N}(q)$ :

$$
R_{N}(q)=\overbrace{\bigcup_{C_{(i, j)}^{N} \in \mathcal{C}_{N}(q)} C_{(i, j)}^{N}}^{0}
$$

With these notations, we can prove the following lemma.

Lemma 2.8. Let $N>1 / \varepsilon$ be a fixed integer. For every $q \in \mathcal{Q}_{\text {ad }}^{\varepsilon}$, the set $\bigcup_{C_{(i, j)}^{N} \in \mathcal{C}_{N}(q)} C_{(i, j)}^{N}$ is a cover of the $\varepsilon$-interior $q^{\varepsilon}$ of $q\left(\right.$ see (1.7)). Moreover, the set $R_{N}(q)$ defined by (2.11) satisfies $q^{\varepsilon} \subset R_{N}(q) \subset q$.

Proof. Let $X \in q^{\varepsilon}$. By definition of $q^{\varepsilon}$, we have $X \in q$ and $d(X, \partial q)>\varepsilon$. Since $\mathbb{R}^{2}$ is covered by squares in $\mathcal{C}_{N}$, there exists $C_{(i, j)}^{N} \in \mathcal{C}_{N}$ such that $X \in C_{(i, j)}^{N}$. Moreover, since $\operatorname{diam}\left(C_{(i, j)}^{N}\right)=\kappa_{N}$, we have $C_{(i, j)}^{N} \subset \bar{B}\left(X, \kappa_{N}\right)$. 


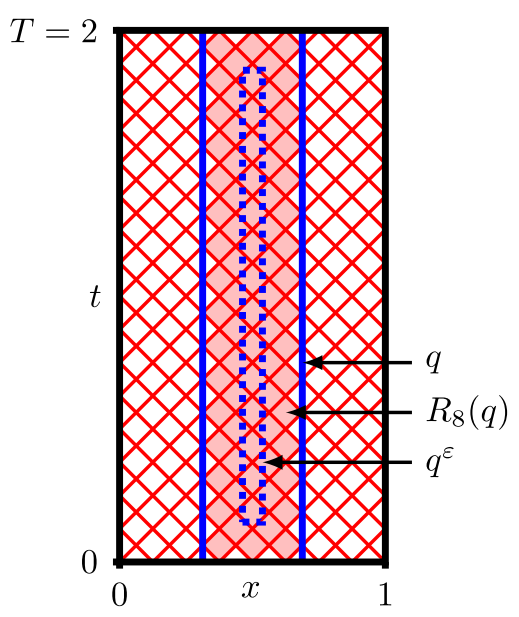

Figure 2. Cover $R_{8}(q)$ of $q^{\varepsilon}$, for $\varepsilon=0.15$.

Let $Y \in \bar{B}\left(X, \kappa_{N}\right)$. Then, for every $Z \in \mathbb{R}^{2} \backslash q$, it follows that

$$
d(Y, Z) \geq|d(Y, X)-d(X, Z)|>\varepsilon-\kappa_{N}>0 .
$$

Consequently, $d\left(Y, \mathbb{R}^{2} \backslash q\right)>0$ which implies $Y \in q$. Therefore, $C_{(i, j)}^{N} \subset \bar{B}\left(X, \kappa_{N}\right) \subset q$ and, finally, $C_{(i, j)}^{N} \in$ $\mathcal{C}_{N}(q)$.

Figure 2 illustrates Lemma 2.8 in the case of the cylindrical observation domain $q=\left(\frac{5}{16}, \frac{11}{16}\right) \times(0,2)$, for $\varepsilon=0.15$ and $N=8$.

Step 3 - Weighted graph associated with $q \in \mathcal{Q}_{\text {ad }}^{\varepsilon}$.

In order to write several expressions in a simpler form, we use elements from graph theory.

More precisely, the introduction of a weighted graph in Definition 2.9 allows to reformulate the observability inequality for the solution $\varphi^{N}$ in terms of a spectral inequality involving the Laplacian matrix of the graph (see Def. 2.11). In this way, the observability constant associated with $\varphi^{N}$ is linked to the first strictly positive eigenvalue of the Laplacian matrix.

Definition 2.9. Let $q \in \mathcal{Q}_{\text {ad }}^{\varepsilon}$ an observation domain. We define the weighted graph $G_{N}(q)$ as follows:

$-\mathbb{I}_{N}$ given by $(2.6)$ is the set of vertices;

- for every $i \in \mathbb{I}_{N}$, the degree of the vertex $i$ is given by:

$$
d_{i}^{N}=\operatorname{Card}\left(\left\{C_{(k,-l)}^{N} \in \mathcal{C}_{N}(q) ; \quad i \in\left\{\mathfrak{j}_{N}(k), \mathfrak{j}_{N}(l)\right\}\right\}\right) ;
$$

- for every $i, j \in \mathbb{I}_{N}$, the weight of the edge linking the vertices $i$ and $j$ is

$$
w_{i, j}^{N}=w_{j, i}^{N}=\operatorname{Card}\left(\left\{C_{(k,-l)}^{N} \in \mathcal{C}_{N}(q) ; \quad\{i, j\}=\left\{\mathfrak{j}_{N}(k), \mathfrak{j}_{N}(l)\right\}\right\}\right) .
$$

Definition 2.10. Let $q \in \mathcal{Q}_{\text {ad }}^{\varepsilon}$ and let $i, j \in \mathbb{I}_{N}$ be two vertices of the graph $G_{N}(q)$. We say that there is a path from $i$ to $j$ in $G_{N}(q)$ and we denote $i \stackrel{N}{\sim} j$ if the vertices $i$ and $j$ are in the same connected component of $G_{N}(q)$. In particular, if $w_{i, j}^{N} \neq 0$, then $i \stackrel{N}{\sim} j$.

We then recall the definition of the Laplacian matrix associated with a graph (see $[4,8]$ ). 
Definition 2.11. Let $q \in \mathcal{Q}_{\text {ad }}^{\varepsilon}$. The Laplacian matrix associated with the graph $G_{N}(q)$ (see Def. 2.9) is the symmetric positive matrix $A_{N}(q) \in \mathcal{M}_{2 N}(\mathbb{R})$ defined by

$$
A_{N}(q)=\left(\begin{array}{cccccc}
d_{-N}^{N} & \cdots & -w_{-N,-1}^{N} & -w_{-N, 1}^{N} & \cdots & -w_{-N, N}^{N} \\
\vdots & \ddots & \vdots & \vdots & & \vdots \\
-w_{-1,-N}^{N} & \cdots & d_{-1}^{N} & -w_{-1,1}^{N} & \cdots & -w_{-1, N}^{N} \\
-w_{1,-N}^{N} & \cdots & -w_{1,-1}^{N} & d_{1}^{N} & \cdots & -w_{1, N}^{N} \\
\vdots & & \vdots & \vdots & \ddots & \vdots \\
-w_{N,-N}^{N} & \cdots & -w_{N,-1}^{N} & -w_{N, 1}^{N} & \cdots & d_{N}^{N}
\end{array}\right)_{2 N \times 2 N}
$$

Remark 2.12. Remark that for every $q \in \mathcal{Q}_{\text {ad }}^{\varepsilon}$, the graph $G_{N}(q)$ has no loop, i.e. $w_{i, i}^{N}=0$ for every $i \in$ $\mathbb{I}_{N}$. Indeed, the elementary squares $C_{(k,-l)}^{N}$ such that $\mathfrak{j}_{N}(k)=\mathfrak{j}_{N}(l)=i$ have their center $x_{(k,-l)}^{N} \in \mathbb{Z}$ and, consequently, cannot be in $Q_{T}$.

Remark also that the Laplacian matrix $A_{N}(q)$ of the graph $G_{N}(q)$ verifies the following property (see $[4,8]$ ): for every $\eta=\left(\eta_{-N}, \ldots, \eta_{-1}, \eta_{1}, \ldots, \eta_{N}\right) \in \mathbb{R}^{2 N}$,

$$
\eta^{T} A_{N}(q) \eta=\sum_{i \in \mathbb{I}_{N}} d_{i}^{N} \eta_{i}^{2}-\sum_{i, j \in \mathbb{I}_{N}} w_{i, j}^{N} \eta_{i} \eta_{j}=\sum_{C_{(i, j)}^{N} \in \mathcal{C}_{N}(q)}\left(\eta_{\mathbf{j}_{N}(i)}-\eta_{-\mathbf{j}_{N}(j)}\right)^{2}
$$

From now on, we consider that the assumption of Lemma 2.8 holds true, i.e. we take $N>1 / \varepsilon$. More precisely, we fix $N$ the smallest integer strictly greater than $1 / \varepsilon$.

Lemma 2.13. Let $q \in \mathcal{Q}_{\mathrm{ad}}^{\varepsilon}$. Then the associated graph $G_{N}(q)$ is connected.

Proof. Let $i \in\{1, \ldots, N-1\}$. We denote by $D_{i}^{+}$the support of the characteristic line " $x+t=x_{i}^{N}$ ", starting from $x_{i}^{N}$ in the direction of decreasing $x$ and following the laws of geometric optics for its reflection on $\Sigma_{T}$. Since $q \in \mathcal{Q}_{\mathrm{ad}}^{\varepsilon}, q^{\varepsilon}$ satisfies the geometric optic condition; consequently, there exists $\left(x^{*}, t^{*}\right) \in q^{\varepsilon} \cap D_{i}^{+}$. From Lemma 2.8, we have $q^{\varepsilon} \subset R_{N}(q)$, so $\left(x^{*}, t^{*}\right)$ belongs to the common side of two elementary squares in $\mathcal{C}_{N}(q)$ :

$$
\left(C_{(k, l)}^{N} \text { and } C_{(k+1, l)}^{N} \text { with } \mathfrak{j}_{N}(k)=i\right) \quad \text { or } \quad\left(C_{(l, k)}^{N} \text { and } C_{(l, k-1)}^{N} \text { with } \mathfrak{j}_{N}(k)=-i\right) .
$$

Therefore $i \stackrel{N}{\sim} i+1$ and, so, the vertices $\{1, \ldots, N\}$ are in the same connected component.

Similarly, we denote by $D_{i}^{-}$the support of the characteristic line " $x-t=x_{i}^{N}$ ", starting from $x_{i}^{N}$ in the direction of increasing $x$ and following the laws of geometric optics for its reflection on $\Sigma_{T}$. Since $q^{\varepsilon}$ satisfies the geometric optic condition, there exists $\left(x^{*}, t^{*}\right) \in q^{\varepsilon} \cap D_{i}^{-}$. From Lemma 2.8, we have $q^{\varepsilon} \subset R_{N}(q)$, so $\left(x^{*}, t^{*}\right)$ belongs to the common side of two elementary squares in $\mathcal{C}_{N}(q)$ :

$$
\left(C_{(l, k)}^{N} \text { and } C_{(l, k+1)}^{N} \text { with } \mathfrak{j}_{N}(k)=i\right) \quad \text { or } \quad\left(C_{(k, l)}^{N} \text { and } C_{(k-1, l)}^{N} \text { with } \mathfrak{j}_{N}(k)=-i\right) .
$$

Therefore $-i \stackrel{N}{\sim}-i-1$ and, so, the vertices $\{-N, \ldots,-1\}$ are in the same connected component.

It remains to show that the vertices $N$ and $-N$ belong to the same connected component. We denote by $D_{N}^{+}$the support of the characteristic line " $x+t=x_{N}^{N}$ ", starting from $x_{N}^{N}$ in the direction of decreasing $x$ and following the laws of geometric optics for its reflection on $\Sigma_{T}$. Since $q^{\varepsilon}$ satisfies the geometric optic condition, there exists $\left(x^{*}, t^{*}\right) \in q^{\varepsilon} \cap D_{N}^{+}$. From Lemma 2.8, we have $q^{\varepsilon} \subset R_{N}(q)$, so $\left(x^{*}, t^{*}\right)$ belongs to the common side of two elementary squares in $\mathcal{C}_{N}(q)$ :

$$
\left(C_{(k, l)}^{N} \text { and } C_{(k+1, l)}^{N} \text { with } \mathfrak{j}_{N}(k)=N\right) \quad \text { or } \quad\left(C_{(l, k)}^{N} \text { and } C_{(l, k-1)}^{N} \text { with } \mathfrak{j}_{N}(k)=-N\right) .
$$


Hence, $N \stackrel{N}{\sim}-N$.

Remark 2.14. A well-known graph theory result (see, for instance, [4], Prop. 1.3.7) states that the graph $G_{N}(q)$ is connected if and only if $\operatorname{dim}\left(\operatorname{ker}\left(A_{N}(q)\right)\right)=1$. Moreover, if $G_{N}(q)$ is connected, then $\operatorname{ker}\left(A_{N}(q)\right)=\operatorname{Vect}\left(\mathbf{1}_{2 N}\right)$, where $\mathbf{1}_{2 N}$ is the vector in $\mathbb{R}^{2 N}$ with all its component equal to 1 .

Let us denote $\lambda_{N}(q)>0$ the smallest non-zero eigenvalue of the matrix $A_{N}(q)$. This eigenvalue is known in graph theory as the algebraic connectivity of the graph. We also define $\lambda_{N}$ by

$$
\lambda_{N}=\min _{q \in \mathcal{Q}_{\text {ad }}^{e}} \lambda_{N}(q)>0 .
$$

Note that since the set $\left\{G_{N}(q) ; q \in \mathcal{Q}_{\mathrm{ad}}^{\varepsilon}\right\}$ has a finite number of elements, $\lambda_{N}$ is well defined.

Step $4-p$-refinement of the graph and connection with the spectrum of the Laplacian matrix.

Definition 2.15. For every $p \in \mathbb{N}^{*}$, we denote by $\mathcal{C}_{N}^{p}(q)$ the set formed by the elementary squares associated with the subdivision $S_{p N}$ and having their interior in $R_{N}(q)$ :

$$
\mathcal{C}_{N}^{p}(q)=\left\{C_{(i, j)}^{p N} \in \mathcal{C}_{p N} ; \quad \stackrel{\circ}{C}_{(i, j)}^{p N} \subset R_{N}(q)\right\} .
$$

We then define the graph $G_{N}^{p}(q)$ following Definition 2.9, substituting $N$ by $p N$ and substituting $\mathcal{C}_{N}(q)$ by $\mathcal{C}_{N}^{p}(q)$ in the definitions of the vertex degrees and the edge weights. Finally, we denote $A_{N}^{p}(q) \in \mathcal{M}_{2 p N}(\mathbb{R})$ the Laplacian matrix associated with the graph $G_{N}^{p}(q)$. This matrix has the following block form:

$$
A_{N}^{p}(q)=\left(\begin{array}{cccccc}
d_{-N}^{N} p I_{p} & \cdots & -w_{-N,-1}^{N} J_{p} & -w_{-N, 1}^{N} J_{p} & \cdots & -w_{-N, N}^{N} J_{p} \\
\vdots & \ddots & \vdots & \vdots & & \vdots \\
-w_{-1,-N}^{N} J_{p} & \cdots & d_{-1}^{N} p I_{p} & -w_{-1,1}^{N} J_{p} & \cdots & -w_{-1, N}^{N} J_{p} \\
-w_{1,-N}^{N} J_{p} & \cdots & -w_{1,-1}^{N} J_{p} & d_{1}^{N} p I_{p} & \cdots & -w_{1, N}^{N} J_{p} \\
\vdots & & \vdots & \vdots & \ddots & \vdots \\
-w_{N,-N}^{N} J_{p} & \cdots & -w_{N,-1}^{N} J_{p} & -w_{N, 1}^{N} J_{p} & \cdots & d_{N}^{N} p I_{p}
\end{array}\right)_{2 p N \times 2 p N}
$$

where $I_{p}, J_{p} \in \mathcal{M}_{p}(\mathbb{R})$ are respectively the identity matrix and the matrix with all its elements equal to 1 .

Definition 2.16. For any $i \in \mathbb{Z}^{*}$, we define the set $\mathbb{J}_{i}^{p}$ by

$$
\mathbb{J}_{i}^{p}= \begin{cases}\{p(i-1)+1, \ldots, p i\} & \text { if } i>0 \\ \{p i, \ldots, p(i+1)-1\} & \text { if } i<0 .\end{cases}
$$

For every $\eta=\left(\eta_{-p N}, \ldots, \eta_{-1}, \eta_{1}, \ldots, \eta_{p N}\right) \in \mathbb{R}^{2 p N}$, we check that

$$
\eta^{T} A_{N}^{p}(q) \eta=\sum_{C_{(i, j)}^{N} \in \mathcal{C}_{N}(q)} \sum_{i^{\prime} \in \mathbb{J}_{i}^{p}} \sum_{j^{\prime} \in \mathbb{J}_{j}^{p}}\left(\eta_{\mathfrak{j}_{p N}\left(i^{\prime}\right)}-\eta_{-\mathfrak{j}_{p N}\left(j^{\prime}\right)}\right)^{2} .
$$

For any $p \in \mathbb{N}^{*}$, the following lemma makes the link between the spectrum of the Laplacian matrix $A_{N}^{p}(q)$ (see Def. 2.15) and the spectrum of the Laplacian matrix $A_{N}(q)$ (see Def. 2.11).

Lemma 2.17. Let $p \in \mathbb{N}^{*}$. The spectrum of the Laplacian matrix $\frac{1}{p} A_{N}^{p}(q)$ (see (2.15)) is composed of the spectrum of the Laplacian matrix $A_{N}(q)$ (see (2.12)), and the diagonal elements of $A_{N}(q)$ repeated $p-1$ times. Moreover, $\operatorname{dim}\left(\operatorname{ker}\left(A_{N}^{p}(q)\right)\right)=1$ and $\operatorname{ker}\left(A_{N}^{p}(q)\right)=\operatorname{Vect}\left(\mathbf{1}_{2 p N}\right)$. 
Proof. Let $\eta=\left(\eta_{-p N}, \ldots, \eta_{-1}, \eta_{1}, \ldots, \eta_{p N}\right) \in \mathbb{R}^{2 p N}$. For every $i \in \mathbb{I}_{N}$, we denote by $\Gamma_{i}=\left(\eta_{i^{\prime}}\right)_{i^{\prime} \in \mathbb{J}_{i}^{p}} \in \mathbb{R}^{p}$ and we group these vectors in the matrix

$$
\Gamma=\left(\Gamma_{-N}|\cdots| \Gamma_{-1}\left|\Gamma_{1}\right| \cdots \mid \Gamma_{N}\right) \in \mathcal{M}_{p, 2 N}(\mathbb{R}) .
$$

In view of $(2.15)$, it follows that

$$
\eta^{T} A_{N}^{p}(q) \eta=p \sum_{i \in \mathbb{I}_{N}} d_{i}^{N} \Gamma_{i}^{T} \Gamma_{i}-\sum_{i, j \in \mathbb{I}_{N}} w_{i, j}^{N} \Gamma_{i}^{T} J_{p} \Gamma_{j}
$$

Since $J_{p}$ is a real symmetric matrix, there exists an orthonormal basis $\left(b_{k}\right)_{1 \leq k \leq p}$ of $\mathbb{R}^{p}$ diagonalizing $J_{p}$. Let us denote $b_{1}=\frac{1}{\sqrt{p}} \mathbf{1}_{p}$. Then, there exists a diagonal matrix $D \in \mathcal{M}_{p}(\mathbb{R})$ and a unitary matrix $Q \in \mathcal{M}_{p}(\mathbb{R})$ such that $J_{p}=Q D Q^{T}$. These matrices have the following form:

$$
D=\left(\begin{array}{cccc}
p & 0 & \cdots & 0 \\
0 & 0 & \cdots & 0 \\
\vdots & \vdots & \ddots & \vdots \\
0 & 0 & \cdots & 0
\end{array}\right)_{p \times p} \text { and } Q=\left(b_{1}|\cdots| b_{p}\right)_{p \times p}
$$

We also define the matrix $U=Q^{T} \Gamma \in \mathcal{M}_{p, 2 N}(\mathbb{R})$, and denote respectively

$$
U_{k, .}=\left(b_{k}^{T} \Gamma_{i}\right)_{i \in \mathbb{I}_{N}} \in \mathbb{R}^{2 N} \quad \text { and } \quad U_{., i}=\left(b_{k}^{T} \Gamma_{i}\right)_{1 \leq k \leq p} \in \mathbb{R}^{p}
$$

the rows and the columns of $U$. Then, for $i, j \in \mathbb{I}_{N}$, we have

$$
\Gamma_{i}^{T} \Gamma_{i}=U_{., i}^{T} U_{., i}=\sum_{k=1}^{p} U_{k, i}^{2} \quad \text { and } \quad \Gamma_{i}^{T} J_{p} \Gamma_{j}=U_{., i}^{T} D U_{., j}=p U_{1, i} U_{1, j}
$$

The spectrum of the matrix $\frac{1}{p} A_{N}^{p}(q)$ can now be computed from

$$
\begin{aligned}
\frac{1}{p} \eta^{T} A_{N}^{p}(q) \eta & =\sum_{i \in \mathbb{I}_{N}} d_{i}^{N} U_{1, i}^{2}-\sum_{i, j \in \mathbb{I}_{N}} w_{i, j}^{N} U_{1, i} U_{1, j}+\sum_{k=2}^{p} \sum_{i \in \mathbb{I}_{N}} d_{i}^{N} U_{k, i}^{2} \\
& =U_{1, .}^{T} A_{N}(q) U_{1, .}+\sum_{k=2}^{p} U_{k, .}^{T} \operatorname{Diag}\left(A_{N}(q)\right) U_{k, .} .
\end{aligned}
$$

Indeed, the expression above shows that $A_{N}^{p}(q)$ is unitarily similar to the block diagonal matrix

$$
\left(\begin{array}{llll}
A_{N}(q) & & & \\
& \operatorname{Diag}\left(A_{N}(q)\right) & & \\
& & \ddots & \\
& & & \operatorname{Diag}\left(A_{N}(q)\right)
\end{array}\right)_{2 p N \times 2 p N} .
$$

Step 5 - Uniform observability for the solution $\varphi^{N}$ associated with $\left(\varphi_{0}^{N}, \varphi_{1}^{N}\right)$ w.r.t. $q \in \mathcal{Q}_{\text {ad }}^{\varepsilon}$. 
Let $N$ be the smallest integer strictly greater than $\varepsilon^{-1}$. We prove an observability inequality for the function $\varphi^{N}$ given by (2.10) and associated with the initial datum $\left(\varphi_{0}^{N}, \varphi_{1}^{N}\right)$ defined in $(2.2)-(2.3)$.

In view of Lemma 2.4 , the function $\left(\varphi_{t}^{N}\right)^{2}$ is constant on each elementary square $C_{(i, j)}^{N}$ in $\mathcal{C}_{N}$ :

$$
\left.\left(\varphi_{t}^{N}\right)^{2}\right|_{C_{(i, j)}^{N}}=\frac{1}{4}\left(\gamma_{\mathbf{j}_{N}(i)}^{N}-\gamma_{-\mathbf{j}_{N}(j)}^{N}\right)^{2}
$$

where $\gamma_{\mathbf{j}_{N}(i)}^{N}$ is given by $(2.7)$ and $\mathfrak{j}_{N}$ by (2.5). In view of the definition of the set $R_{N}(q)$ (see $(2.11)$ ), we can estimate from below the $L^{2}(q)$-norm of $\varphi_{t}^{N}$ as follows:

$$
\begin{aligned}
\iint_{q}\left(\varphi_{t}^{N}\right)^{2} \geq \iint_{R_{N}(q)}\left(\varphi_{t}^{N}\right)^{2} & =\sum_{C_{(i, j)}^{N} \in \mathcal{C}_{N}(q)} \iint_{C_{(i, j)}^{N}}\left(\varphi_{t}^{N}\right)^{2} \\
& =\frac{1}{8 N^{2}} \sum_{C_{(i, j)}^{N} \in \mathcal{C}_{N}(q)}\left(\gamma_{\mathbf{j}_{N}(i)}^{N}-\gamma_{-\mathbf{j}_{N}(j)}^{N}\right)^{2} .
\end{aligned}
$$

In the previous equality, we used that the area of every elementary square in $\mathcal{C}_{N}$ is equal to $\frac{1}{2 N^{2}}$. Combining (2.17) with the relation (2.13), we obtain

$$
\iint_{q}\left(\varphi_{t}^{N}\right)^{2} \geq \frac{1}{8 N^{2}}\left(\gamma^{N}\right)^{T} A_{N}(q) \gamma^{N}
$$

with $\gamma^{N}=\left(\gamma_{-N}^{N}, \ldots, \gamma_{-1}^{N}, \gamma_{1}^{N}, \ldots, \gamma_{N}^{N}\right) \in \mathbb{R}^{2 N}$ and $\gamma_{i}^{N}$ given by $(2.7)$.

It is easy to see that $\gamma^{N} \in \operatorname{ker}\left(A_{N}(q)\right)^{\perp}$. Indeed, applying Lemma 2.13, the graph $G_{N}(q)$ is connected. Then, from Remark 2.14, we have $\operatorname{ker}\left(A_{N}(q)\right)=\operatorname{Vect}\left(\mathbf{1}_{2 N}\right)$ and, since $\alpha_{i}^{N}=\alpha_{-i}^{N}$ and $\beta_{i}^{N}=-\beta_{-i}^{N}$, the vector $\gamma^{N}$ verifies

$$
\left(\gamma^{N}\right)^{T} \mathbf{1}_{2 N}=\sum_{i \in \mathbb{I}_{N}} \gamma_{i}^{N}=2 \sum_{i=1}^{N} \alpha_{i}^{N}=0 .
$$

Then, using $\lambda_{N}$ defined in (2.14), it follows that

$$
\begin{aligned}
\left(\gamma^{N}\right)^{T} A_{N}(q) \gamma^{N} \geq \lambda_{N} \sum_{i \in \mathbb{I}_{N}}\left(\gamma_{i}^{N}\right)^{2} & =2 \lambda_{N} \sum_{i=1}^{N}\left(\left(\alpha_{i}^{N}\right)^{2}+\left(\beta_{i}^{N}\right)^{2}\right) \\
& =2 N \lambda_{N}\left(\left\|\varphi_{0}^{N}\right\|_{H_{0}^{1}(\Omega)}^{2}+\left\|\varphi_{1}^{N}\right\|_{L^{2}(\Omega)}^{2}\right) .
\end{aligned}
$$

From (2.18) and (2.19), we deduce the following observability inequality:

$$
\left\|\left(\varphi_{0}^{N}, \varphi_{1}^{N}\right)\right\|_{\mathbf{V}}^{2} \leq \frac{4 N}{\lambda_{N}}\left\|\varphi_{t}^{N}\right\|_{L^{2}(q)}^{2}
$$

where the constant $\frac{4 N}{\lambda_{N}}$ is independent of the domain $q$ and the initial datum $\left(\varphi_{0}, \varphi_{1}\right)$.

Step 6 - Uniform observability for the solution $\varphi^{p N}$ associated with $\left(\varphi_{0}^{p N}, \varphi_{1}^{p N}\right)$ w.r.t. $q \in \mathcal{Q}_{\text {ad }}^{\varepsilon}$ and $p \in \mathbb{N}^{*}$.

The next step consists in obtaining a uniform observability inequality for an initial datum $\left(\varphi_{0}^{p N}, \varphi_{1}^{p N}\right)$ of the form (2.2)-(2.3) with respect to $q \in \mathcal{Q}_{\text {ad }}^{\varepsilon}$ and $p \in \mathbb{N}^{*}$. As in the previous step, we get that $\left(\varphi_{t}^{p N}\right)^{2}$ is constant 
on every elementary square $C_{\left(i^{\prime}, j^{\prime}\right)}^{p N} \in \mathcal{C}_{p N}$ :

$$
\left.\left(\varphi_{t}^{p N}\right)^{2}\right|_{C_{\left(i^{\prime}, j^{\prime}\right)}^{p N}}=\frac{1}{4}\left(\gamma_{\mathfrak{j}_{p N}\left(i^{\prime}\right)}^{p N}-\gamma_{-\mathfrak{j}_{p N}\left(j^{\prime}\right)}^{p N}\right)^{2}
$$

where $\varphi^{p N}$ is the solution of (1.3) associated with the initial datum $\left(\varphi_{0}^{p N}, \varphi_{1}^{p N}\right)$ given by $(2.2)-(2.3)$, and $\gamma_{\mathbf{j}_{p N}}^{p N}\left(^{\prime}\right)$ is defined by (2.7).

Then, remark that every elementary square $C_{(i, j)}^{N} \in \mathcal{C}_{N}$ is the union of $p^{2}$ elementary squares in $\mathcal{C}_{p N}$, or more precisely that

$$
C_{(i, j)}^{N}=\bigcup_{i^{\prime} \in \mathbb{J}_{i}^{p}} \bigcup_{j^{\prime} \in \mathbb{J}_{j}^{p}} C_{\left(i^{\prime}, j^{\prime}\right)}^{p N}, \quad \forall i, j \in \mathbb{Z}^{*}
$$

Using the above expression in the evaluation of the $L^{2}(q)$-norm of $\varphi_{t}^{p N}$, we have

$$
\begin{aligned}
\iint_{q}\left(\varphi_{t}^{p N}\right)^{2} & \geq \iint_{R_{N}(q)}\left(\varphi_{t}^{p N}\right)^{2}=\sum_{C_{(i, j)}^{N} \in \mathcal{C}_{N}(q)} \iint_{C_{(i, j)}^{N}}\left(\varphi_{t}^{p N}\right)^{2} \\
& =\sum_{C_{(i, j)}^{N} \in \mathcal{C}_{N}(q)} \sum_{i^{\prime} \in \mathbb{J}_{i}^{p}} \sum_{j^{\prime} \in \mathbb{J}_{j}^{p}} \iint_{C_{\left(i^{\prime}, j^{\prime}\right)}^{p N}}\left(\varphi_{t}^{p N}\right)^{2} \\
& =\frac{1}{8 p^{2} N^{2}} \sum_{C_{(i, j)}^{N} \in \mathcal{C}_{N}(q)} \sum_{i^{\prime} \in \mathbb{J}_{i}^{p}} \sum_{j^{\prime} \in \mathbb{J}_{j}^{p}}\left(\gamma_{\mathfrak{j}_{p N}}^{p N}-\gamma_{\left.-i_{p N}\right)}^{p N}\left(j^{\prime}\right)\right)^{2} \\
& =\frac{1}{8 p^{2} N^{2}}\left(\gamma^{p N}\right)^{T} A_{N}^{p}(q) \gamma^{p N} .
\end{aligned}
$$

Since $G_{N}(q)$ is a connected graph, the degree $d_{i}^{N}$ of every vertex $i \in \mathbb{I}_{N}$ verifies $d_{i}^{N} \geq 1$. Applying Lemma 2.17, the smallest non-zero eigenvalue $\lambda_{N}^{p}(q)$ of $\frac{1}{p} A_{N}^{p}(q)$ verifies

$$
\lambda_{N}^{p}(q)=\min \left(\lambda_{N}(q), \min _{i \in \mathbb{I}_{N}} d_{i}^{N}\right) \geq \min \left(\lambda_{N}, 1\right)>0 .
$$

The vector $\gamma^{p N}=\left(\gamma_{i^{\prime}}^{p N}\right)_{i^{\prime} \in \mathbb{I}_{p N}}$ belongs to $\operatorname{ker}\left(A_{N}^{p}(q)\right)^{\perp}$. Indeed,

$$
\left(\gamma^{p N}\right)^{T} \mathbf{1}_{2 p N}=\sum_{i^{\prime} \in \mathbb{I}_{p N}} \gamma_{i^{\prime}}^{p N}=2 \sum_{i^{\prime}=1}^{p N} \alpha_{i^{\prime}}^{p N}=0 .
$$

Setting $\widehat{\lambda}_{N}=\min \left(\lambda_{N}, 1\right)$, it follows that

$$
\begin{aligned}
\frac{1}{p}\left(\gamma^{p N}\right)^{T} A_{N}^{p}(q) \gamma^{p N} \geq \widehat{\lambda}_{N} \sum_{i^{\prime} \in \mathbb{I}_{p N}}\left(\gamma_{i^{\prime}}^{p N}\right)^{2} & =2 \widehat{\lambda}_{N} \sum_{i^{\prime}=1}^{p N}\left(\left(\alpha_{i^{\prime}}^{p N}\right)^{2}+\left(\beta_{i^{\prime}}^{p N}\right)^{2}\right) \\
& =2 p N \widehat{\lambda}_{N}\left(\left\|\varphi_{0}^{p N}\right\|_{H_{0}^{1}(\Omega)}^{2}+\left\|\varphi_{1}^{p N}\right\|_{L^{2}(\Omega)}^{2}\right) .
\end{aligned}
$$


Consequently, combining the above relation with (2.20), we obtain the following observability inequality:

$$
\left\|\left(\varphi_{0}^{p N}, \varphi_{1}^{p N}\right)\right\|_{\mathbf{V}}^{2} \leq \frac{4 N}{\widehat{\lambda}_{N}}\left\|\varphi_{t}^{p N}\right\|_{L^{2}(q)}^{2}
$$

where the constant $\frac{4 N}{\widehat{\lambda}_{N}}$ is independent of the domain $q$, the initial datum $\left(\varphi_{0}, \varphi_{1}\right)$ and the integer $p$.

Step 7 - Passing to the limit $p \rightarrow \infty$ and conclusion.

In order to finish the proof, we pass to the limit when $p \rightarrow \infty$ in the observability inequality (2.21). We easily check that as $p$ tends to $\infty$,

$$
\varphi_{0}^{p N} \rightarrow \varphi_{0} \quad \text { in } H_{0}^{1}(\Omega) \quad \text { and } \quad \varphi_{1}^{p N} \rightarrow \varphi_{1} \quad \text { in } L^{2}(\Omega)
$$

Moreover, since the solution $\varphi$ of the wave equation (1.3) depends continuously on its initial condition $\left(\varphi_{0}, \varphi_{1}\right) \in$ $\mathbf{V}$, we can write

$$
\varphi_{t}^{p N} \rightarrow \varphi_{t} \quad \text { in } L^{2}\left(0, T ; L^{2}(\Omega)\right)
$$

Eventually, passing to the limit in (2.21), we get

$$
\left\|\left(\varphi_{0}, \varphi_{1}\right)\right\|_{\mathbf{V}}^{2} \leq \max \left\{4 N, \frac{4 N}{\lambda_{N}}\right\}\left\|\varphi_{t}\right\|_{L^{2}(q)}^{2}, \quad \forall\left(\varphi_{0}, \varphi_{1}\right) \in \mathbf{V}
$$

which concludes the proof with $C_{\mathrm{obs}}^{\varepsilon}=\max \left\{4 N, \frac{4 N}{\lambda_{N}}\right\}$. We recall that $N$ depends on $\varepsilon$ by the condition $N>1 / \varepsilon$.

Remark 2.18. Let $q \subset Q_{T}$ be a finite union of open sets. If $q$ verifies the usual geometric optic condition, there exists $\varepsilon>0$ small enough such that $q^{\varepsilon}$ still verifies the geometric optic condition. We then set $N=\lfloor 1 / \varepsilon\rfloor+1$. The associated graph $G_{N}(q)$ being connected, there exists a relation (see, for instance, [22]) between the algebraic connectivity $\lambda_{N}(q)$, the number of vertices $N_{V}$ and the diameter $D_{G}$ of the graph. More precisely, we have $\lambda_{N}(q) \geq \frac{4}{N_{V} D_{G}}$. Since in our case $N_{V}=2 N$ and $D_{G} \leq 2 N$, we deduce that $\lambda_{N}(q) \geq \frac{1}{N^{2}}$ and therefore that $C_{\text {obs }}(q) \leq 4 N^{3}$. Thus, in the worst situation, we could have an observability constant of order $1 / \varepsilon^{3}$. If we see $\varepsilon$ as a measure of the "thickness" of the observation domain $q$, we get the same estimate of the observability constant as the one given in Proposition 2.1 of [29].

\subsection{One explicit example}

We illustrate the proof of Theorem 2.1 on a simple example for which the observation domain $q$ depicted in Figure 3 (colored in red) is well adapted to the subdivision $S_{4}$. The study of this example is also the opportunity to develop a method for the computation of the observability constant for observation domains which are exactly the union of elementary squares associated with a given subdivision $S_{N}$, for a fixed integer $N>0$.

We start by enumerating the elementary squares composing the observation domain $q$. In Table 1 , we list, for $i, j \in \mathbb{Z}^{*}$, the elementary squares $C_{(i, j)}^{4}$ included in $q$ and the values of the indices $\mathfrak{j}_{4}(i)$ and $-\mathfrak{j}_{4}(j)$, allowing to compute the Laplacian matrix $A_{4}(q)$ associated with the corresponding graph $G_{4}(q)$. 


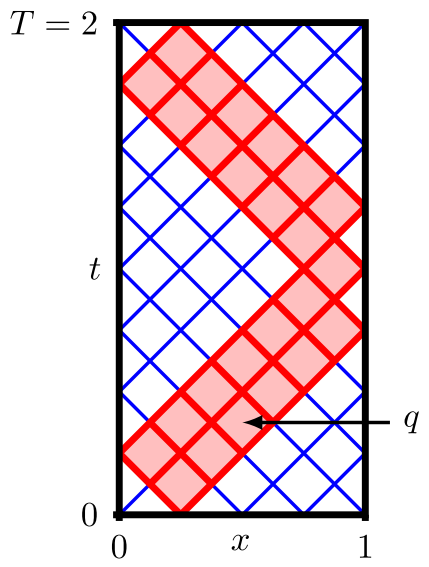

Figure 3. Observation domain $q$ adapted to $S_{4}$.

TABLE 1 . Elementary squares associated with $S_{4}$ and belonging to $\mathcal{C}_{4}(q)$.

\begin{tabular}{lcclccccc}
\hline$C_{(i, j)}^{4}$ & $\mathfrak{j}_{4}(i)$ & $-\mathfrak{j}_{4}(j)$ & $C_{(i, j)}^{4}$ & $\mathfrak{j}_{4}(i)$ & $-\mathfrak{j}_{4}(j)$ & $C_{(i, j)}^{4}$ & $\mathfrak{j}_{4}(i)$ & $-\mathfrak{j}_{4}(j)$ \\
\hline$C_{(2,1)}^{4}$ & 2 & -1 & $C_{(6,-1)}^{4}$ & -3 & 1 & $C_{(9,-4)}^{4}$ & 1 & 4 \\
$C_{(2,-1)}^{4}$ & 2 & 1 & $C_{(7,1)}^{4}$ & -2 & -1 & $C_{(8,-5)}^{4}$ & -1 & -4 \\
$C_{(3,1)}^{4}$ & 3 & -1 & $C_{(7,-1)}^{4}$ & -2 & 1 & $C_{(9,-5)}^{4}$ & 1 & -4 \\
$C_{(3,-1)}^{4}$ & 3 & 1 & $C_{(8,-1)}^{4}$ & -1 & 1 & $C_{(8,-6)}^{4}$ & -1 & -3 \\
$C_{(4,1)}^{4}$ & 4 & -1 & $C_{(8,-2)}^{4}$ & -1 & 2 & $C_{(9,-6)}^{4}$ & 1 & -3 \\
$C_{(4,-1)}^{4}$ & 4 & 1 & $C_{(9,-2)}^{4}$ & 1 & 2 & $C_{(8,-7)}^{4}$ & -1 & -2 \\
$C_{(5,1)}^{4}$ & -4 & -1 & $C_{(8,-3)}^{4}$ & -1 & 3 & $C_{(9,-7)}^{4}$ & 1 & -2 \\
$C_{(5,-1)}^{4}$ & -4 & 1 & $C_{(9,-3)}^{4}$ & 1 & 3 & & & \\
$C_{(6,1)}^{4}$ & -3 & -1 & $C_{(8,-4)}^{4}$ & -1 & 4 & & & \\
\hline
\end{tabular}

The Laplacian matrix associated with the graph $G_{4}(q)$ is given by

$$
A_{4}(q)=\left(\begin{array}{cccccccc}
4 & 0 & 0 & -2 & -2 & 0 & 0 & 0 \\
0 & 4 & 0 & -2 & -2 & 0 & 0 & 0 \\
0 & 0 & 4 & -2 & -2 & 0 & 0 & 0 \\
-2 & -2 & -2 & 13 & -1 & -2 & -2 & -2 \\
-2 & -2 & -2 & -1 & 13 & -2 & -2 & -2 \\
0 & 0 & 0 & -2 & -2 & 4 & 0 & 0 \\
0 & 0 & 0 & -2 & -2 & 0 & 4 & 0 \\
0 & 0 & 0 & -2 & -2 & 0 & 0 & 4
\end{array}\right)_{8 \times 8}
$$

The spectrum of $A_{4}(q)$ can be explicitly computed:

$$
\operatorname{Sp}\left(A_{4}(q)\right)=\{0,4,4,4,4,4,14,16\}
$$

It confirms that the kernel of $A_{4}(q)$ is one-dimensional - therefore $G_{4}(q)$ is connected - and implies that the smallest non-zero eigenvalue of $A_{4}(q)$ is $\lambda_{4}(q)=4$. If we replace the subdivision $S_{4}$ by the subdivision $S_{4 p}$ for 
any $p \in \mathbb{N}^{*}$, then the Laplacian matrix associated with the graph $G_{4}^{p}(q)$ is the following one:

$$
A_{4}^{p}(q)=\left(\begin{array}{cccccccc}
4 p I_{p} & 0_{p} & 0_{p} & -2 J_{p} & -2 J_{p} & 0_{p} & 0_{p} & 0_{p} \\
0_{p} & 4 p I_{p} & 0_{p} & -2 J_{p} & -2 J_{p} & 0_{p} & 0_{p} & 0_{p} \\
0_{p} & 0_{p} & 4 p I_{p} & -2 J_{p} & -2 J_{p} & 0_{p} & 0_{p} & 0_{p} \\
-2 J_{p} & -2 J_{p} & -2 J_{p} & 13 p I_{p} & -J_{p} & -2 J_{p} & -2 J_{p} & -2 J_{p} \\
-2 J_{p} & -2 J_{p} & -2 J_{p} & -J_{p} & 13 p I_{p} & -2 J_{p} & -2 J_{p} & -2 J_{p} \\
0_{p} & 0_{p} & 0_{p} & -2 J_{p} & -2 J_{p} & 4 p I_{p} & 0_{p} & 0_{p} \\
0_{p} & 0_{p} & 0_{p} & -2 J_{p} & -2 J_{p} & 0_{p} & 4 p I_{p} & 0_{p} \\
0_{p} & 0_{p} & 0_{p} & -2 J_{p} & -2 J_{p} & 0_{p} & 0_{p} & 4 p I_{p}
\end{array}\right)_{8 p \times 8 p}
$$

According to Lemma 2.17, the smallest non-zero eigenvalue of $\frac{1}{p} A_{4}^{p}(q)$ is given by

$$
\lambda_{4}^{p}(q)=\min \left(\lambda_{4}(q), \min _{i \in \mathbb{I}_{4}} d_{i}^{4}\right)=\min (4,4)=4
$$

Consequently, the observability constant associated with the observation domain $q$ depicted in Figure 3 is given by

$$
C_{\mathrm{obs}}(q)=\frac{4 \cdot 4}{\lambda_{4}^{p}(q)}=4
$$

\subsection{A corollary}

We prove a uniform observability inequality for the observation domains $q_{\gamma}$ defined in (1.9), with $\gamma \in \mathcal{G}_{\text {ad }}$ (see (1.10)), which will be used in the next section.

Corollary 2.19. Let $T \geq 2$. There exists a constant $C_{\mathrm{obs}}>0$ such that for every $\gamma \in \mathcal{G}_{\mathrm{ad}}$,

$$
\left\|\left(\varphi_{0}, \varphi_{1}\right)\right\|_{\mathbf{W}}^{2} \leq C_{\mathrm{obs}}\|\varphi\|_{L^{2}\left(q_{\gamma}\right)}^{2}, \quad \forall\left(\varphi_{0}, \varphi_{1}\right) \in \mathbf{W},
$$

where $\varphi$ is the solution of the wave equation (1.3) associated with the initial condition $\left(\varphi_{0}, \varphi_{1}\right)$.

Proof. We show that for any $\varepsilon>0$ small enough, $\left\{q_{\gamma} ; \gamma \in \mathcal{G}_{\text {ad }}\right\} \subset \mathcal{Q}_{\text {ad }}^{\varepsilon}$. Let $\gamma \in \mathcal{G}_{\text {ad }}$. We introduce the sets $\Gamma_{ \pm}=\left\{\left(\gamma(t) \pm \delta_{0}, t\right) ; t \in[0, T]\right\}, \widetilde{\Gamma}_{ \pm}=\left\{\left(\gamma(t) \pm \frac{\delta_{0}}{2}, t\right) ; t \in[0, T]\right\}$ and $Q_{T}^{\varepsilon}=\Omega \times(\varepsilon, T-\varepsilon) \cdot \gamma$ being a $M$-Lipschitz curve, we can show that

$$
d\left(\widetilde{\Gamma}_{ \pm}, \Gamma_{ \pm}\right) \geq \frac{\delta_{0}}{2 \sqrt{M^{2}+1}}
$$

Then, for $\varepsilon<\frac{\delta_{0}}{2 \sqrt{M^{2}+1}}$, we have $\widetilde{q}_{\gamma} \cap Q_{T}^{\varepsilon} \subset q_{\gamma}^{\varepsilon}$, with the observation domain $\widetilde{q}_{\gamma}$ defined as in (1.9) with a half-width of $\delta_{0} / 2$. The domain $\widetilde{q}_{\gamma} \cap Q_{T}^{\varepsilon}$ verifies the geometric optic condition because $\delta_{0} \leq \gamma \leq 1-\delta_{0}$ and $T-2 \varepsilon \geq 2\left(1-\delta_{0}\right)$. Consequently, $q_{\gamma}^{\varepsilon}$ also verifies the geometric optic condition and $q_{\gamma} \in \mathcal{Q}_{\text {ad }}^{\varepsilon}$. We conclude the proof by noticing that the constant $\frac{\delta_{0}}{2 \sqrt{M^{2}+1}}$ is independent of the choice of $\gamma$.

Finally, the observability inequality follows from Theorem 2.1 with $C_{\text {obs }}=C_{\text {obs }}^{\varepsilon}$, for any $\varepsilon<\frac{\delta_{0}}{2 \sqrt{M^{2}+1}}$. We conclude the proof by noticing that the constant $\frac{\delta_{0}}{2 \sqrt{M^{2}+1}}$ is independent of the choice of $\gamma$.

We end this section by emphasizing the role of the bound $M$ on the derivative of the curve $\gamma$ introduced in the set $\mathcal{G}_{\text {ad }}$, namely $\left\|\gamma^{\prime}\right\|_{L^{\infty}(0, T)} \leq M$. As can be seen in the previous proof, if $M$ goes to infinity, then $\varepsilon$ goes to zero, and consequently, in view of Remark 2.18, the constant $C_{\mathrm{obs}}^{\varepsilon}$ blows up. This is illustrated in Figure 4 where a ray starting from the point $\left(\frac{1}{2}, 0\right)$ only intersects the control region on a narrow part corresponding to 


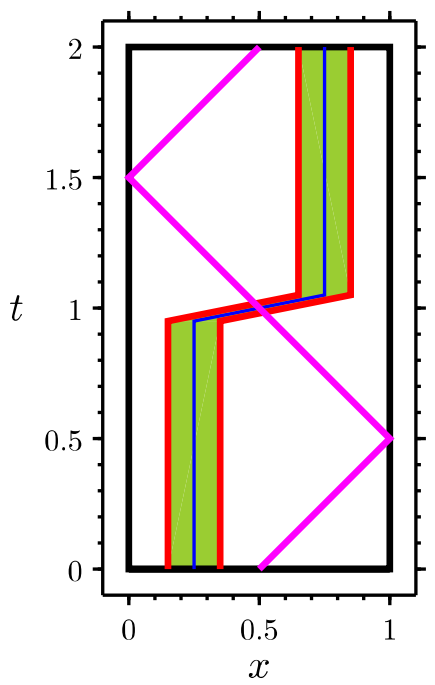

FiguRE 4. Ray intersecting a narrow part (in the neighborhood of the point $(1 / 2,1)$ ) of the control region corresponding to a large slope of $\gamma$.

a large slope of $\gamma$. Any initial condition generating a solution localized around such ray leads to an arbitrarily large cost of control. The constraint on the slope of $\gamma$ has been introduced to avoid this phenomenon.

\section{Optimization of THE ShAPE OF THE CONTROL DOMAin}

In this section, we study the problem of finding the optimal shape and position of the control domain, for a given initial condition $\left(y_{0}, y_{1}\right) \in \mathbf{V}$.

\subsection{Existence of an optimal domain}

In order to obtain a well-posedness result, we consider a variant of the optimal problem (1.11) and replace the characteristic function $\mathbb{1}_{q}$ in (1.1) by a regular function in space. More precisely, we fix $\delta \in\left(0, \delta_{0}\right)$ and, for every $\gamma \in \mathcal{G}_{\text {ad }}$, we define $\chi_{\gamma}(x, t)=\chi(x-\gamma(t))$, with $\chi: \mathbb{R} \rightarrow[0,1]$ a $C^{1}$ even function such that

$$
\chi(x)= \begin{cases}1 & \text { if } x \in\left(-\delta_{0}+\delta, \delta_{0}-\delta\right), \\ 0 & \text { if } x \notin\left(-\delta_{0}, \delta_{0}\right), \\ \in(0,1) & \text { otherwise. }\end{cases}
$$

In the sequel, we also use the function $\chi_{\gamma}^{\prime}$ defined by $\chi_{\gamma}^{\prime}(x, t)=\chi^{\prime}(x-\gamma(t))$. In this new setting, the HUM control now lives in the weighted space

$$
L_{\chi}^{2}\left(q_{\gamma}\right):=L^{2}\left(q_{\gamma} ; \chi_{\gamma}\right)=\left\{v: q_{\gamma} \rightarrow \mathbb{R} ; \iint_{q_{\gamma}} v^{2} \chi_{\gamma}<+\infty\right\} .
$$

Moreover, we can adapt the uniform observability inequality given in Corollary 2.19. For $T \geq 2$, there exists a constant $C_{\text {obs }}>0$ such that for every $\gamma \in \mathcal{G}_{\text {ad }}$,

$$
\left\|\left(\varphi_{0}, \varphi_{1}\right)\right\|_{\mathbf{W}}^{2} \leq C_{\mathrm{obs}}\|\varphi\|_{L_{\chi}^{2}\left(q_{\gamma}\right)}^{2}, \quad \forall\left(\varphi_{0}, \varphi_{1}\right) \in \mathbf{W},
$$

where $\varphi$ is the solution of (1.3) associated with the initial condition $\left(\varphi_{0}, \varphi_{1}\right)$. 
Then, our optimization problem reads as follows: for a given initial datum $\left(y_{0}, y_{1}\right) \in \mathbf{V}$, solve

$$
\inf _{\gamma \in \mathcal{G}_{\mathrm{ad}}} J(\gamma)=\|v\|_{L_{\chi}^{2}\left(q_{\gamma}\right)}^{2}=\iint_{q_{\gamma}} \varphi^{2} \chi_{\gamma},
$$

where $v$ is the control of minimal $L_{\chi}^{2}$-norm distributed over $q_{\gamma} \subset Q_{T}$, and $\varphi$ is the associated adjoint state such that $v=\left.\varphi\right|_{q_{\gamma}}$. This adjoint state can be characterized using the HUM method, it is the solution of (1.3) associated with the minimum $\left(\varphi_{0}, \varphi_{1}\right)$ of the conjugate functional

$$
\mathcal{J}_{\gamma}^{\star}\left(\varphi_{0}, \varphi_{1}\right)=\frac{1}{2} \iint_{q_{\gamma}} \varphi^{2} \chi_{\gamma}-\left\langle\varphi_{1}, y_{0}\right\rangle_{H^{-1}(\Omega), H_{0}^{1}(\Omega)}+\left\langle\varphi_{0}, y_{1}\right\rangle_{L^{2}(\Omega)}, \quad \forall\left(\varphi_{0}, \varphi_{1}\right) \in \mathbf{W} .
$$

To show the well-posedness of (3.3), we follow the steps of Theorem 2.1 in [29]. We start with a convergence result on the function $\chi_{\gamma}$.

Lemma 3.1. Let $\left(\gamma_{n}\right)_{n \geq 0} \subset \mathcal{G}_{\text {ad }}$ and $\gamma \in \mathcal{G}_{\text {ad }}$. If $\gamma_{n} \rightarrow \gamma$ in $L^{\infty}(0, T)$, then $\chi_{\gamma_{n}} \rightarrow \chi_{\gamma}$ in $L^{\infty}\left(Q_{T}\right)$.

Proof. It is a direct consequence of the inequality $\left\|\chi_{\gamma_{n}}-\chi_{\gamma}\right\|_{L^{\infty}\left(Q_{T}\right)} \leq\left\|\chi^{\prime}\right\|_{L^{\infty}(\mathbb{R})}\left\|\gamma_{n}-\gamma\right\|_{L^{\infty}(0, T)}$.

We then have that the following continuity result.

Proposition 3.2. The cost $J$ is continuous over $\mathcal{G}_{\text {ad }}$ for the norm $L^{\infty}(0, T)$.

Proof. Let $\left(\gamma_{n}\right)_{n \geq 0} \subset \mathcal{G}_{\text {ad }}$ and $\gamma \in \mathcal{G}_{\text {ad }}$ such that $\gamma_{n} \rightarrow \gamma$ in $L^{\infty}(0, T)$ as $n \rightarrow \infty$.

For any $n \in \mathbb{N}$, we denote $\left(\varphi_{0}^{n}, \varphi_{1}^{n}\right) \in \mathbf{W}$ the minimum of $\mathcal{J}_{\gamma_{n}}^{\star}$, and $\varphi^{n}$ the corresponding solution of (1.3).

Using the uniform observability inequality (3.2) and the optimality condition of $\mathcal{J}_{\gamma_{n}}^{\star}$, it follows that

$$
\begin{aligned}
\left\|\left(\varphi_{0}^{n}, \varphi_{1}^{n}\right)\right\|_{\mathbf{W}}^{2} \leq C_{\mathrm{obs}} \iint_{q_{\gamma_{n}}}\left(\varphi^{n}\right)^{2} \chi_{\gamma_{n}} & =C_{\mathrm{obs}}\left(\left\langle\varphi_{1}^{n}, y_{0}\right\rangle_{H^{-1}, H_{0}^{1}}-\left\langle\varphi_{0}^{n}, y_{1}\right\rangle_{L^{2}}\right) \\
& \leq C_{\mathrm{obs}}\left\|\left(\varphi_{0}^{n}, \varphi_{1}^{n}\right)\right\|_{\mathbf{W}}\left\|\left(y_{0}, y_{1}\right)\right\|_{\mathbf{V}}
\end{aligned}
$$

leading to the uniform bound $\left\|\left(\varphi_{0}^{n}, \varphi_{1}^{n}\right)\right\|_{\mathbf{w}} \leq C_{\mathrm{obs}}\left\|\left(y_{0}, y_{1}\right)\right\|_{\mathbf{v}}$. Consequently, there exist two functions $\varphi_{0} \in$ $L^{2}(\Omega)$ and $\varphi_{1} \in H^{-1}(\Omega)$ such that, up to a subsequence, as $n \rightarrow \infty$

$$
\varphi_{0}^{n} \rightarrow \varphi_{0} \text { weakly in } L^{2}(\Omega) \text { and } \varphi_{1}^{n} \rightarrow \varphi_{1} \text { weakly in } H^{-1}(\Omega) .
$$

From the continuous dependence of the solution of the wave equation with respect to the initial condition, it follows

$$
\varphi^{n} \rightarrow \varphi \quad \text { weakly in } L^{2}\left(0, T ; L^{2}(\Omega)\right),
$$

where $\varphi$ is the solution of (1.3) associated with $\left(\varphi_{0}, \varphi_{1}\right)$.

Let $\psi \in L^{2}\left(0, T ; L^{2}(\Omega)\right)$. We then have

$$
\iint_{Q_{T}} \psi \varphi^{n} \chi_{\gamma_{n}}=\iint_{Q_{T}} \psi \varphi^{n} \chi_{\gamma}+\iint_{Q_{T}} \psi \varphi^{n}\left(\chi_{\gamma_{n}}-\chi_{\gamma}\right) \rightarrow \iint_{Q_{T}} \psi \varphi \chi_{\gamma} .
$$

Indeed, we can take the weak limit in the first term because $\psi \chi_{\gamma} \in L^{2}\left(0, T ; L^{2}(\Omega)\right)$. Using Lemma 3.1 and the boundedness of $\left(\varphi^{n}\right)_{n \geq 0}$ in $L^{2}\left(0, T ; L^{2}(\Omega)\right)$, the second term converges to 0 in view of the estimate

$$
\left|\iint_{Q_{T}} \psi \varphi^{n}\left(\chi_{\gamma_{n}}-\chi_{\gamma}\right)\right| \leq\|\psi\|_{L^{2}\left(L^{2}\right)}\left\|\varphi^{n}\right\|_{L^{2}\left(L^{2}\right)}\left\|\chi_{\gamma_{n}}-\chi_{\gamma}\right\|_{L^{\infty}\left(Q_{T}\right)}
$$


Consequently, we obtain the convergence

$$
\varphi^{n} \chi_{\gamma_{n}} \rightarrow \varphi \chi_{\gamma} \quad \text { weakly in } L^{2}\left(0, T ; L^{2}(\Omega)\right) \text {. }
$$

Let now $\left(\psi_{0}, \psi_{1}\right) \in \mathbf{W}$ and $\psi$ the corresponding solution of (1.3). Taking the weak limit in the optimality condition

$$
\iint_{q_{\gamma_{n}}} \psi \varphi^{n} \chi_{\gamma_{n}}=\left\langle\psi_{1}, y_{0}\right\rangle_{H^{-1}, H_{0}^{1}}-\int_{\Omega} \psi_{0} y_{1}
$$

we find

$$
\iint_{q_{\gamma}} \psi \varphi \chi_{\gamma}=\left\langle\psi_{1}, y_{0}\right\rangle_{H^{-1}, H_{0}^{1}}-\int_{\Omega} \psi_{0} y_{1}
$$

This means that $\left(\varphi_{0}, \varphi_{1}\right)$ is the minimum of $\mathcal{J}_{\gamma}^{\star}$. Besides, we remark that this property uniquely characterizes the weak limit of any subsequence of $\left(\varphi_{0}^{n}, \varphi_{1}^{n}\right)$. This implies that the whole sequence $\left(\varphi_{0}^{n}, \varphi_{1}^{n}\right)$ weakly converges. The continuity of $J$ is finally obtained by taking the weak limit in the optimality condition

$$
\iint_{q_{\gamma_{n}}}\left(\varphi^{n}\right)^{2} \chi_{\gamma_{n}}=\left\langle\varphi_{1}^{n}, y_{0}\right\rangle_{H^{-1}, H_{0}^{1}}-\int_{\Omega} \varphi_{0}^{n} y_{1} \rightarrow\left\langle\varphi_{1}, y_{0}\right\rangle_{H^{-1}, H_{0}^{1}}-\int_{\Omega} \varphi_{0} y_{1}=\iint_{q_{\gamma}} \varphi^{2} \chi_{\gamma}
$$

The continuity of $J$ then allows to show that the extremal problem (3.3) is well-posed.

Proposition 3.3. The cost $J$ reaches its minimum over $\mathcal{G}_{\text {ad }}$.

Proof. The cost $J$ being bounded from below, there exists a minimizing sequence $\left(\gamma_{n}\right)_{n \geq 0} \subset \mathcal{G}_{\text {ad }}$. By definition of $\mathcal{G}_{\text {ad }}$, this sequence is bounded in $W^{1, \infty}(0, T)$. Moreover, since $W^{1, \infty}(0, T)$ is compactly embedded in $L^{\infty}(0, T)$, there exists a curve $\gamma \in L^{\infty}(0, T)$ such that, up to a subsequence, $\gamma_{n} \rightarrow \gamma$ in $L^{\infty}(0, T)$. From the definition of $\mathcal{G}_{\text {ad }}$, all the curves $\gamma_{n}$ are $M$-Lipschitz, with $M$ independent of $n$. So, taking the pointwise limit in the expressions

$$
\begin{array}{ll}
\left|\gamma_{n}(t)-\gamma_{n}(s)\right| \leq M|t-s|, & \forall t, s \in[0, T], \\
\delta_{0} \leq \gamma_{n}(t) \leq 1-\delta_{0}, & \forall t \in[0, T],
\end{array}
$$

we notice that $\gamma \in \mathcal{G}_{\text {ad }}$. Finally, using Proposition 3.2, we obtain $J\left(\gamma_{n}\right) \rightarrow J(\gamma)=\inf _{\mathcal{G}_{\text {ad }}} J$ which means that $\gamma$ is a minimum of $J$ over $\mathcal{G}_{\text {ad }}$.

It follows in particular from Proposition 3.2 that the optimal cost $J\left(\gamma^{\star}\right)=\inf _{\gamma \in \mathcal{G}_{\text {ad }}} J(\gamma)$ is stable with respect to the initial datum $\left(y_{0}, y_{1}\right) \in \mathbf{V}$ in the following sense: $J\left(\gamma^{\star}\right) \leq C_{\text {obs }}\left\|\left(y_{0}, y_{1}\right)\right\|_{\mathbf{V}}^{2}$, where $C_{\text {obs }}$ is the observability constant appearing in Corollary 2.19. However, we highlight that the optimal curve $\gamma^{\star}$ does not vary continuously with respect to $\left(y_{0}, y_{1}\right)$ (see Sect. 4.1).

Remark 3.4. We emphasize that the functional $J$ may have several global minima. Indeed, if the initial datum $\left(y_{0}, y_{1}\right) \in \mathbf{V}$ is such that $y_{0}(x)=-y_{0}(1-x), y_{1}(x)=0$ for $x \in \Omega$, there are examples (see [29] and example (EX1) in Sect. 4.1) where the optimal domain is not the cylinder centered at $x=\frac{1}{2}$. Hence, if $\gamma^{\star}$ is an optimal curve for $J$ corresponding to this initial datum, from symmetry arguments, the curve $1-\gamma^{\star}$ is also optimal. 


\subsection{First directional derivative of the cost $J$}

We now give the expression of the directional derivative of the cost $J$.

Definition 3.5. Let $\gamma, \bar{\gamma} \in W^{1, \infty}(0, T)$, with $\delta_{0} \leq \gamma \leq 1-\delta_{0}$. The perturbation $\bar{\gamma}$ is said admissible if and only if for any $\eta>0$ small enough, the perturbed curve $\gamma_{\eta}=\gamma+\eta \bar{\gamma}$ verifies $\delta_{0} \leq \gamma_{\eta} \leq 1-\delta_{0}$.

Lemma 3.6. Let $\chi \in C^{2}(\mathbb{R})$ and $\gamma, \bar{\gamma} \in W^{1, \infty}(0, T)$, with $\delta_{0} \leq \gamma \leq 1-\delta_{0}$. For any $\eta>0$, we define the perturbed curve $\gamma_{\eta}=\gamma+\eta \bar{\gamma}$. Taking $\eta \rightarrow 0$, we then have

$$
\frac{\chi_{\gamma_{\eta}}-\chi_{\gamma}}{\eta} \rightarrow-\bar{\gamma} \chi_{\gamma}^{\prime} \quad \text { in } L^{\infty}\left(Q_{T}\right)
$$

Proof. It is a direct consequence the inequality

$$
\left\|\chi_{\gamma_{\eta}}-\chi_{\gamma}+\eta \bar{\gamma} \chi_{\gamma}^{\prime}\right\|_{L^{\infty}\left(Q_{T}\right)} \leq \frac{\eta^{2}}{2}\left\|\chi^{\prime \prime}\right\|_{L^{\infty}(\mathbb{R})}\|\bar{\gamma}\|_{L^{\infty}(0, T)}^{2}
$$

Proposition 3.7. Let $\chi \in C^{2}(\mathbb{R})$ and $\gamma, \bar{\gamma} \in W^{1, \infty}(0, T)$, with $\delta_{0} \leq \gamma \leq 1-\delta_{0}$. For any $\eta>0$, we define the perturbed curve $\gamma_{\eta}=\gamma+\eta \bar{\gamma}$. If $\bar{\gamma}$ is an admissible perturbation, then the directional derivative of $J$ at $\gamma$ in the direction $\bar{\gamma}$, denoted by $d J(\gamma ; \bar{\gamma})$, reads

$$
d J(\gamma ; \bar{\gamma})=\lim _{\eta \rightarrow 0} \frac{J\left(\gamma_{\eta}\right)-J(\gamma)}{\eta}=\int_{0}^{T} \bar{\gamma} \int_{\Omega} \varphi^{2} \chi_{\gamma}^{\prime}
$$

where $\varphi$ is the solution of (1.3) associated with the minimum $\left(\varphi_{0}, \varphi_{1}\right)$ of $\mathcal{J}_{\gamma}^{\star}$.

Proof. For $\eta>0$ small enough, we denote by $\left(\varphi_{0}^{\eta}, \varphi_{1}^{\eta}\right)$ the minimum of $\mathcal{J}_{\gamma_{\eta}}^{\star}$, and $\varphi^{\eta}$ the corresponding solution of (1.3). Similarly, we denote by $\left(\varphi_{0}, \varphi_{1}\right)$ the minimum of $\mathcal{J}_{\gamma}^{\star}$, and $\varphi$ the corresponding solution of (1.3). Using the optimality conditions of $\mathcal{J}_{\gamma_{\eta}}^{\star}$ and $\mathcal{J}_{\gamma}^{\star}$, we can write

$$
\begin{aligned}
J\left(\gamma_{\eta}\right)-J(\gamma) & =\iint_{q_{\gamma_{\eta}}}\left(\varphi^{\eta}\right)^{2} \chi_{\gamma_{\eta}}-\iint_{q_{\gamma}} \varphi^{2} \chi_{\gamma} \\
& =\left(\left\langle\varphi_{1}^{\eta}, y_{0}\right\rangle_{H^{-1}, H_{0}^{1}}-\int_{\Omega} \varphi_{0}^{\eta} y_{1}\right)-\left(\left\langle\varphi_{1}, y_{0}\right\rangle_{H^{-1}, H_{0}^{1}}-\int_{\Omega} \varphi_{0} y_{1}\right) \\
& =\iint_{q_{\gamma}} \varphi^{\eta} \varphi \chi_{\gamma}-\iint_{q_{\gamma_{\eta}}} \varphi \varphi^{\eta} \chi_{\gamma_{\eta}}=-\iint_{Q_{T}} \varphi^{\eta} \varphi\left(\chi_{\gamma_{\eta}}-\chi_{\gamma}\right) .
\end{aligned}
$$

Arguing as in the proof of Proposition 3.2, we can show that $\varphi^{\eta} \rightarrow \varphi$ weakly in $L^{2}\left(0, T ; L^{2}(\Omega)\right)$. As a result, we have

$$
\begin{aligned}
\frac{J\left(\gamma_{\eta}\right)-J(\gamma)}{\eta} & =\iint_{Q_{T}} \varphi^{\eta} \varphi \bar{\gamma} \chi_{\gamma}^{\prime}-\iint_{Q_{T}} \varphi^{\eta} \varphi\left(\frac{\chi_{\gamma_{\eta}}-\chi_{\gamma}}{\eta}+\bar{\gamma} \chi_{\gamma}^{\prime}\right) \\
& \rightarrow \iint_{Q_{T}} \varphi^{2} \bar{\gamma} \chi_{\gamma}^{\prime}=\int_{0}^{T} \bar{\gamma} \int_{\Omega} \varphi^{2} \chi_{\gamma}^{\prime} .
\end{aligned}
$$


Indeed, we can take the weak limit in the first term since $\varphi \bar{\gamma} \chi_{\gamma}^{\prime} \in L^{2}\left(0, T ; L^{2}(\Omega)\right)$. Moreover, using Lemma 3.6 and the boundedness of $\left(\varphi^{\eta}\right)_{\eta>0}$ in $L^{2}\left(0, T ; L^{2}(\Omega)\right)$, the second term converges to 0 in view of the estimate

$$
\left|\iint_{Q_{T}} \varphi^{\eta} \varphi\left(\frac{\chi_{\gamma_{\eta}}-\chi_{\gamma}}{\eta}+\bar{\gamma} \chi_{\gamma}^{\prime}\right)\right| \leq\left\|\varphi^{\eta}\right\|_{L^{2}\left(L^{2}\right)}\|\varphi\|_{L^{2}\left(L^{2}\right)}\left\|\frac{\chi_{\gamma_{\eta}}-\chi_{\gamma}}{\eta}+\bar{\gamma} \chi_{\gamma}^{\prime}\right\|_{L^{\infty}\left(Q_{T}\right)} .
$$

Remark 3.8. We emphasize that the expression of the directional derivative $\mathrm{d} J(\gamma ; \bar{\gamma})$ does not involve the solution of an adjoint boundary value problem. This is due to the fact that the curve $\gamma$, argument of the cost $J$, is associated with the control of minimal $L^{2}\left(q_{\gamma}\right)$-norm. We refer to the proof of Theorem 2.3 in [23] for more details.

\subsection{Regularization and Gradient algorithm}

At the practical level, in order to solve the optimal problem (3.3) numerically, we need to handle the Lipschitz constraint included in $\mathcal{G}_{\text {ad }}$. In this respect, we add a regularizing term to the cost $J$ in order to keep the derivative of $\gamma$ uniformly bounded. The optimization problem is now the following one: for $\epsilon>0$ fixed, solve

$$
\min _{\substack{\gamma \in W^{1, \infty}(0, T) \\ \delta_{0} \leq \gamma \leq 1-\delta_{0}}} J_{\epsilon}(\gamma)=J(\gamma)+\frac{\epsilon}{2}\left\|\gamma^{\prime}\right\|_{L^{2}(0, T)}^{2} .
$$

The regularization parameter $\epsilon$, which can be compared to the Lipschitz constant $M$ in the definition of $\mathcal{G}_{\text {ad }}$, controls the speed of variation of the curves $\gamma \in W^{1, \infty}(0, T)$.

We fix $\gamma \in W^{1, \infty}(0, T)$ such that $\delta_{0} \leq \gamma \leq 1-\delta_{0}$. Using Proposition 3.7, for any admissible perturbation $\bar{\gamma} \in W^{1, \infty}(0, T)$, a direct calculation provides the expression of the directional derivative of $J_{\epsilon}$

$$
\mathrm{d} J_{\epsilon}(\gamma ; \bar{\gamma})=\mathrm{d} J(\gamma ; \bar{\gamma})+\epsilon \int_{0}^{T} \gamma^{\prime} \bar{\gamma}^{\prime}=\left\langle j_{\gamma}, \bar{\gamma}\right\rangle_{L^{2}(0, T)}+\epsilon\left\langle\gamma^{\prime}, \bar{\gamma}^{\prime}\right\rangle_{L^{2}(0, T)}
$$

with

$$
j_{\gamma}(t)=\int_{\Omega} \varphi^{2}(x, t) \chi_{\gamma}^{\prime}(x, t) \mathrm{d} x, \quad \forall t \in[0, T]
$$

In the expression of $j_{\gamma}$, the function $\varphi$ is the solution of (1.3) associated with the minimum $\left(\varphi_{0}, \varphi_{1}\right)$ of $\mathcal{J}_{\gamma}^{\star}$. Consequently, a minimizing sequence $\left(\gamma_{n}\right)_{n \in \mathbb{N}}$ for $J_{\epsilon}$ is defined as follows:

$$
\left\{\begin{array}{l}
\gamma_{0} \text { given in } H^{1}(0, T) \\
\gamma_{n+1}=P_{\left[\delta_{0}, 1-\delta_{0}\right]}\left(\gamma_{n}-\rho j_{\gamma_{n}}^{\epsilon}\right), \quad \text { for } n \geq 0
\end{array}\right.
$$

where $P_{\left[\delta_{0}, 1-\delta_{0}\right]}$ is the pointwise projection in the interval $\left[\delta_{0}, 1-\delta_{0}\right], \rho>0$ a descent step-size and $j_{\gamma_{n}}^{\epsilon} \in H^{1}(0, T)$ is the solution of the variational formulation

$$
\left\langle j_{\gamma_{n}}^{\epsilon}, \widetilde{\gamma}\right\rangle_{L^{2}(0, T)}+\epsilon\left\langle j_{\gamma_{n}}^{\epsilon \prime}, \widetilde{\gamma}^{\prime}\right\rangle_{L^{2}(0, T)}=\left\langle j_{\gamma_{n}}, \widetilde{\gamma}\right\rangle_{L^{2}(0, T)}+\epsilon\left\langle\gamma_{n}^{\prime}, \widetilde{\gamma}^{\prime}\right\rangle_{L^{2}(0, T)}, \quad \forall \widetilde{\gamma} \in H^{1}(0, T) .
$$

This implies that $\mathrm{d} J_{\epsilon}\left(\gamma_{n} ; j_{\gamma_{n}}^{\epsilon}\right)=\left\|j_{\gamma_{n}}^{\epsilon}\right\|_{L^{2}(0, T)}^{2}+\epsilon\left\|j_{\gamma_{n}}^{\epsilon \prime}\right\|_{L^{2}(0, T)}^{2} \geq 0$.

Remark 3.9. For simplicity, we have relaxed here the constraint $\left\|\gamma^{\prime}\right\|_{L^{\infty}(0, T)} \leq M$ appearing in the set $\mathcal{G}_{\text {ad }}$ (see (1.10)) through the regularization term $\frac{\epsilon}{2}\left\|\gamma^{\prime}\right\|_{L^{2}(0, T)}^{2}$, with $\epsilon>0$. We may instead consider the term $\frac{\epsilon}{2}\left\|\gamma^{\prime \prime}\right\|_{L^{2}(0, T)}^{2}$ 
ensuring that $\left\|\gamma^{\prime}\right\|_{L^{\infty}(0, T)} \leq M_{\epsilon}$, with $M_{\epsilon}=\mathcal{O}\left(\epsilon^{-1}\right)$. Our numerical experiments - some of them discussed in the next section - suggest that this later possibility is not necessary.

\section{NumERICAL EXPERIMENTS}

Before presenting some numerical experiments, let us briefly mention some aspects of the resolution of the underlying discretized problem.

- The discretization of the curve $\gamma$ is performed as follows. For any fixed integer $\mathcal{N}>0$, we denote $\delta t=T / \mathcal{N}$ and define the uniform subdivision $\left\{t_{i}\right\}_{i=0, \cdots, \mathcal{N}}$ of $[0, T]$ with $t_{i}=i \delta t$. We then approximate the curve $\gamma$ in the space of dimension $\mathcal{N}+1$

$$
\mathbb{P}_{1}^{\delta t}=\left\{\gamma \in C([0, T]) ;\left.\gamma\right|_{\left[t_{i-1}, t_{i}\right]} \text { affine, } \forall i \in\{1, \ldots, \mathcal{N}\}\right\}
$$

For any $\gamma \in \mathbb{P}_{1}^{\delta t}, \gamma=\sum_{i=0}^{\mathcal{N}} \gamma^{i} L_{i}^{\delta t}$ with $\left(\gamma^{i}\right)_{0 \leq i \leq \mathcal{N}} \in \Omega^{\mathcal{N}+1}$ where $\left(L_{i}^{\delta t}\right)_{0 \leq i \leq \mathcal{N}}$ is the usual Lagrange basis. Consequently, $\gamma$ is defined by the $\mathcal{N}+1$ points $\left(\gamma^{i}, t_{i}\right) \in \Omega \times[0, T]$. The knowledge of the initial curve $\gamma_{0} \in \mathbb{P}_{1}^{\delta t}$ such that $\delta_{0} \leq \gamma_{0} \leq 1-\delta_{0}$ determines such points and then a triangular mesh of $Q_{T}$. At each iteration $n \geq 0$, these points are updated along the $x$-axis according to the pointwise time descent direction $j_{\gamma_{n}}^{\epsilon} \in H^{1}(0, T)$ (see $(3.5))$ as follows:

$$
x_{i}^{n+1}=P_{\left[\delta_{0}, 1-\delta_{0}\right]}\left(x_{i}^{n}-\rho j_{\gamma_{n}}^{\epsilon}\left(t_{i}^{n}\right)\right), \quad t_{i}^{n+1}=t_{i}^{n}, \quad \forall i=0, \cdots, \mathcal{N}+1
$$

We emphasize that a re-meshing of $Q_{T}$ is performed at each iteration $n$ according to the set of points $\left(x_{i}^{n}, t_{i}^{n}\right)_{i=0, \cdots, \mathcal{N}+1}$.

Remark that, for a fixed value of $\mathcal{N}$, the curves $\gamma \in \mathbb{P}_{1}^{\delta t}$ are uniformly Lipschitz with constant $M=\frac{1}{\delta t}$, as required in $\mathcal{G}_{\text {ad }}$.

- Each iteration of the algorithm requires the numerical approximation of the control of minimal $L^{2}\left(q_{\gamma_{n}}\right)$-norm for the initial datum $\left(y_{0}, y_{1}\right) \in \mathbf{V}$. We use the space-time method described in Sections 3-4 of [6], which is very well-adapted to the description of $\gamma$ embedded in a space-time mesh of $Q_{T}$. The minimization of the conjugate functional $\mathcal{J}_{\gamma_{n}}^{\star}$ (see (3.4)) with respect to $\left(\varphi_{0}, \varphi_{1}\right) \in \mathbf{W}$ is replaced by the search of the unique saddle-point of the Lagrangian $\mathcal{L}: \mathbf{Z} \times L^{2}\left(0, T ; H_{0}^{1}(\Omega)\right) \rightarrow \mathbb{R}$ defined by

$$
\mathcal{L}(\varphi, y)=\frac{1}{2}\|\varphi\|_{L^{2}\left(q_{\gamma_{n}}\right)}^{2}-\int_{0}^{T}\langle L \varphi, y\rangle_{H^{-1}(\Omega), H_{0}^{1}(\Omega)}+\left\langle\varphi_{t}(\cdot, 0), y_{0}\right\rangle_{H^{-1}(\Omega), H_{0}^{1}(\Omega)}-\left\langle\varphi(\cdot, 0), y_{1}\right\rangle_{L^{2}(\Omega)},
$$

with $\mathbf{Z}=C\left([0, T] ; L^{2}(\Omega)\right) \cap C^{1}\left([0, T] ; H^{-1}(\Omega)\right)$. The corresponding mixed formulation is solved with a conformal space-time finite element method, while a direct method is used to invert the discrete matrix. The interesting feature of the method, for which the adaptation of the mesh is very simple to handle, is that only a small part of the matrix - corresponding to the term $\|\varphi\|_{L^{2}\left(q_{\gamma_{n}}\right)}^{2}$ - is modified between two consecutive iterations $n$ and $n+1$. Moreover, this space-time framework leads to strong convergent approximations of the control as the fineness of the mesh goes to zero. We refer to [6] for more details.

\subsection{Numerical illustrations}

We discuss several experiments performed with FreeFEM++ (see [14]) for various initial data and control domains. We notably use an UMFPACK type solver.

We denote in this section by $J_{\epsilon}^{n}$ and $\mathrm{d} J_{\epsilon}^{n}$ a numerical approximation of $J_{\epsilon}\left(\gamma_{n}\right)$ and $\mathrm{d} J_{\epsilon}\left(\gamma_{n}\right)$ respectively. 
TABLE 2. (EX1) - Number of iterations, optimal value of the functional $J_{\varepsilon}$ and performance index, for the initial curves $\left(\gamma_{0}^{i}\right)_{i \in\{1,2,3\}}$ given by (4.1).

\begin{tabular}{cccc}
\hline Initial curve & $\gamma_{0}^{1}$ & $\gamma_{0}^{2}$ & $\gamma_{0}^{3}$ \\
\hline Number of iterations & 33 & 33 & 84 \\
$J_{\epsilon}\left(\gamma_{\text {opt }}\right)$ & 47.09 & 47.09 & 47.93 \\
$\Pi\left(\gamma_{\text {opt }}\right)$ & $-0.32 \%$ & $-0.32 \%$ & $-2.11 \%$ \\
\hline
\end{tabular}

We fix $\delta_{0}=0.15$ and $\delta=\delta_{0} / 4$. Moreover, according to (3.1), we define the function $\chi \in C^{2}(\mathbb{R})$ in $\left[\delta_{0}-\delta, \delta_{0}\right]$ as the unique polynomial of degree 5 such that $\chi\left(\delta_{0}-\delta\right)=1, \chi\left(\delta_{0}\right)=\chi^{\prime}\left(\delta_{0}-\delta\right)=\chi^{\prime}\left(\delta_{0}\right)=\chi^{\prime \prime}\left(\delta_{0}-\delta\right)=\chi^{\prime \prime}\left(\delta_{0}\right)=0$ and vanishing on $\mathbb{R} \backslash\left[\delta_{0}-\delta, \delta_{0}\right]$.

Concerning the stopping criterion for the descent algorithm, we observed that the usual one based on the relative quantity $\left|J_{\epsilon}^{n}-J_{\epsilon}^{n-1}\right| / J_{\epsilon}^{0}$ is inefficient because too noisy. This is due to the uncertainty on the numerical computation of the adjoint state $\varphi^{n}$ and the perturbation $j_{\gamma_{n}}^{\epsilon}$. Consequently, in order to better capture the variations of the sequence $\left(J_{\epsilon}^{n}\right)_{n \in \mathbb{N}}$, we replace $J_{\epsilon}^{n}$ and $J_{\epsilon}^{n-1}$ by the right and left $p$-point average respectively leading to the stopping criterion

$$
\Delta J_{\epsilon}^{n}:=\left|\frac{\frac{1}{p} \sum_{i=n}^{n+p-1} J_{\epsilon}^{i}-\frac{1}{p} \sum_{i=n-p}^{n-1} J_{\epsilon}^{i}}{J_{\epsilon}^{0}}\right|<\eta, \quad \text { for } p \in \mathbb{N}^{*} \text { fixed. }
$$

In the sequel, we fix $p=10$ and $\eta=10^{-3}$.

Furthermore, in order to measure the gain obtained by using non-cylindrical control domains rather than cylindrical ones, we introduce a performance index associated with each optimal curves $\gamma_{\text {opt }}$ : identifying any constant curve $\gamma \equiv x_{0}$ with its value $x_{0} \in\left[\delta_{0}, 1-\delta_{0}\right]$, we compute the minimal cost $\min _{x_{0}} J_{\epsilon}\left(x_{0}\right)$ for cylindrical domains; the performance index of $\gamma_{\mathrm{opt}}$ is then defined as follows:

$$
\Pi\left(\gamma_{\mathrm{opt}}\right)=100\left(1-\frac{J_{\epsilon}\left(\gamma_{\mathrm{opt}}\right)}{\min _{x_{0}} J_{\epsilon}\left(x_{0}\right)}\right) .
$$

In the sequel, in practice, the minimum of $J_{\epsilon}$ with respect to $x_{0}$ is searched among 13 distinct values equi-distributed between 0.2 and 0.8 .

- We first consider the regular initial datum $\left(y_{0}, y_{1}\right)$ given by

$$
y_{0}(x)=\sin (2 \pi x), \quad y_{1}(x)=0, \quad \text { for } x \in(0,1)
$$

and $T=2, \epsilon=10^{-2}, \rho=10^{-4}$. We initialize the descent algorithm with the following three initial curves:

$$
\gamma_{0}^{1}(t)=\frac{2}{5}, \quad \gamma_{0}^{2}(t)=\frac{3}{5}, \quad \gamma_{0}^{3}(t)=\frac{1}{2}+\frac{1}{10} \cos \left(\pi \frac{t}{T}\right), \quad \text { for } t \in(0, T)
$$

The corresponding initial and optimal domains are depicted in Figure 5 together with typical space-time meshes. The numbers of iterations until convergence, the values of the functional $J_{\varepsilon}$ evaluated at the optimal curve $\gamma_{\text {opt }}$ and the performance indices of $\gamma_{\text {opt }}$ are listed in Table 2.

In Figure 5, we observe that the optimal domain computed by the algorithm depends on the chosen initial domain. This indicates that the functional $J_{\varepsilon}$ have several local minima. Moreover, one can show that, among the cylindrical domains, there are two optimal values, $x_{0}=1 / 4$ and $x_{0}=3 / 4$, leading to $J_{\varepsilon}\left(x_{0}\right) \approx 46.94$. These values correspond to the extrema of the function $x \rightarrow \sin (2 \pi x), x \in[0,1]$. The simulations associated with 

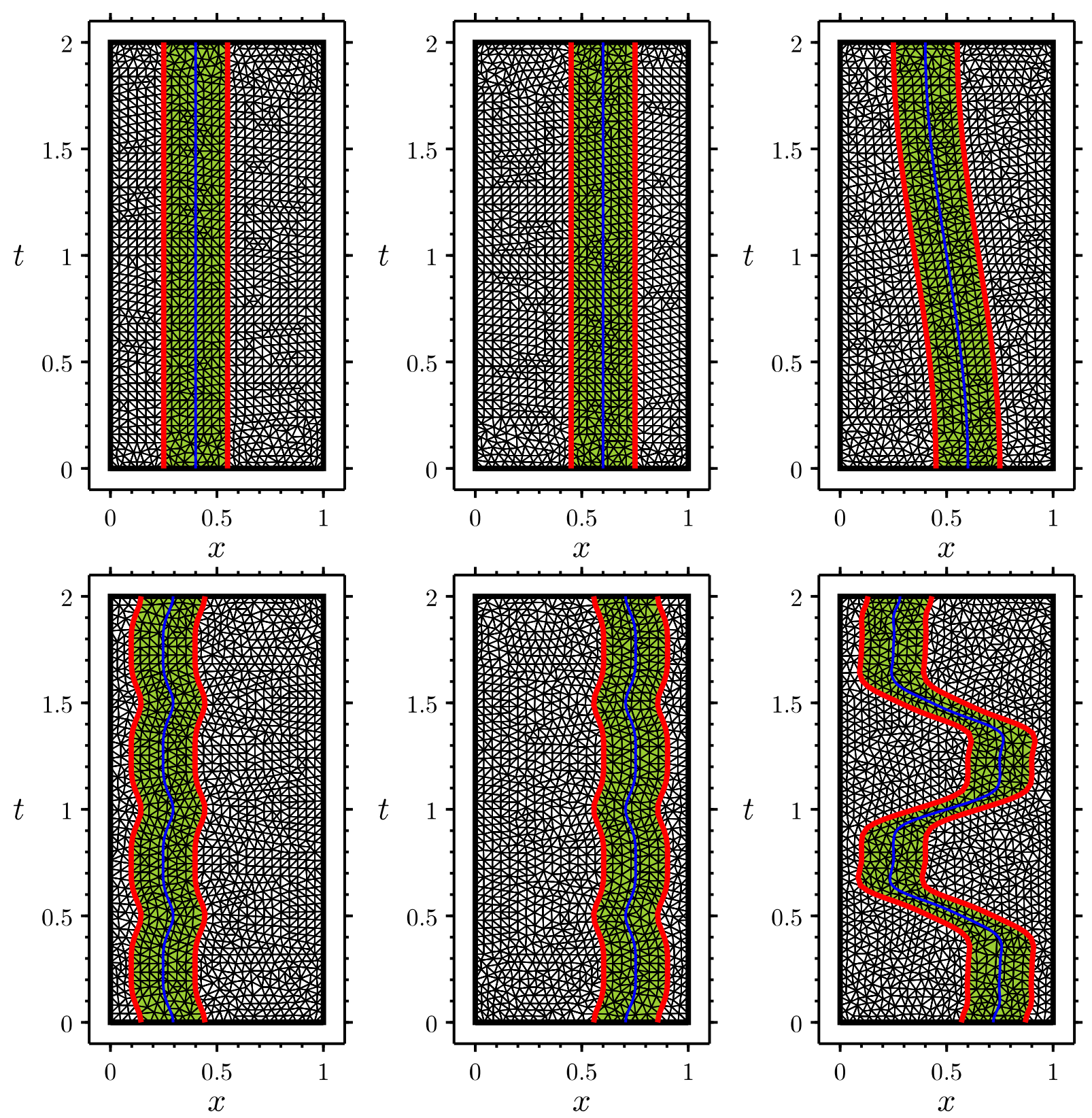

Figure 5. (EX1) - Initial top and optimal (bottom) control domains for the initial curves $\left(\gamma_{0}^{i}\right)_{i \in\{1,2,3\}}$ given by (4.1) (from left to right).

the initial curves $\gamma_{0}^{1}$ and $\gamma_{0}^{2}$ are in agreement with this fact. On the other hand, the worst cylindrical domain corresponds to $x_{0}=1 / 2$ (see Fig. 7-Left).

Eventually, the adjoint states $\varphi$ (from which we obtain the control $v=\left.\varphi\right|_{q_{\gamma}^{n}}$ ) computed for the optimal domains in Figure 5-Bottom, are displayed in Figure 6. 

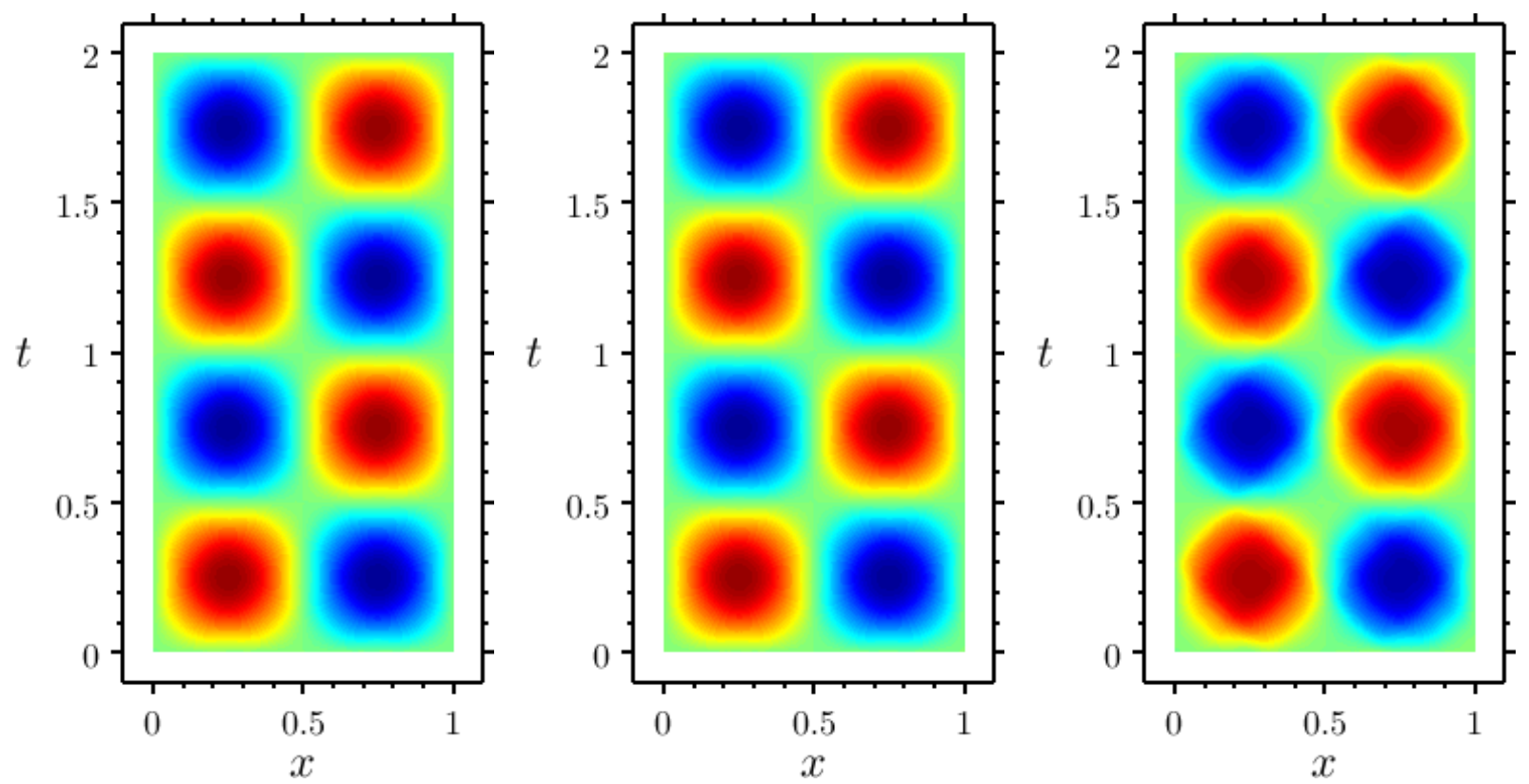

Figure 6. (EX1) - Isovalues of the adjoint states $\varphi$ computed for the optimal domains obtained for the initial curves $\left(\gamma_{0}^{i}\right)_{i \in\{1,2,3\}}$ given by (4.1) (from left to right) and for the initial datum (EX1).
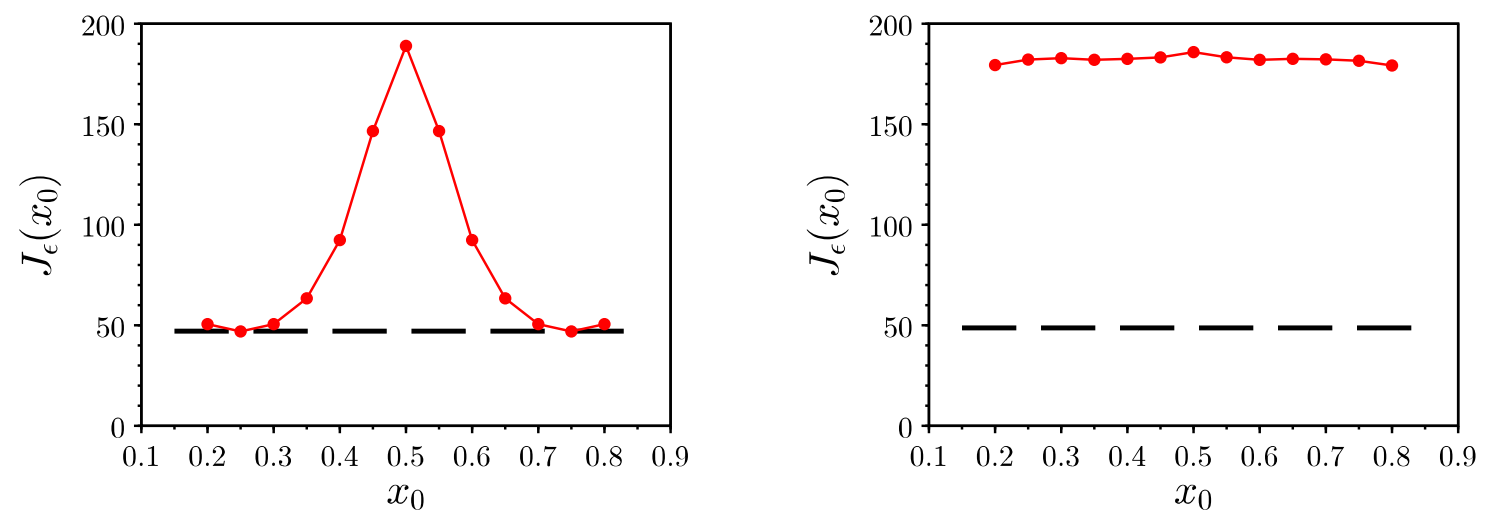

FiguRE 7 . Values of $J_{\epsilon}$ for constant curves $\gamma \equiv x_{0}(\bullet)$, for the initial datum (EX1) (left) and (EX2) (right). The dashed line (- -) represents the value of $J_{\epsilon}\left(\gamma_{\text {opt }}\right)$, for the initial curves $\gamma_{0} \equiv 2 / 5$ (left) and $\gamma_{0} \equiv 1 / 2$ (right).

- We now consider the initial datum $\left(y_{0}, y_{1}\right)$ given by

$$
y_{0}(x)=(10 x-4)^{2}(10 x-6)^{2} \mathbb{1}_{[0.4,0.6]}(x), \quad y_{1}(x)=y_{0}^{\prime}(x), \quad \text { for } x \in(0,1) .
$$

As can be seen in Figure 9-3, this initial condition, plotted in Figure 8, generates a travelling wave.

For $T=2, \epsilon=10^{-2}$ and $\rho=10^{-4}$, we initialize the descent algorithm with the curve $\gamma_{0} \equiv 1 / 2$. The convergence is reached after 68 iterations and the optimal cost is $J_{\epsilon}\left(\gamma_{\text {opt }}\right) \approx 48.70$. Moreover, the minimal cost 

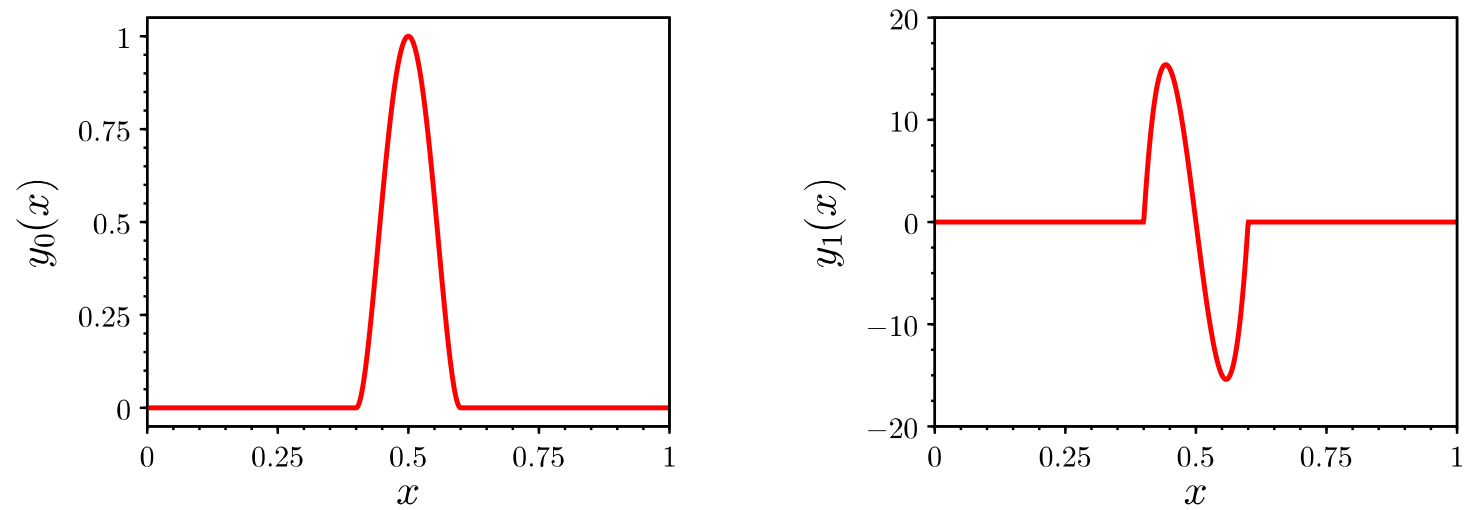

FIgURE 8. Initial datum $\left(y_{0}, y_{1}\right)$ defined in (EX2).
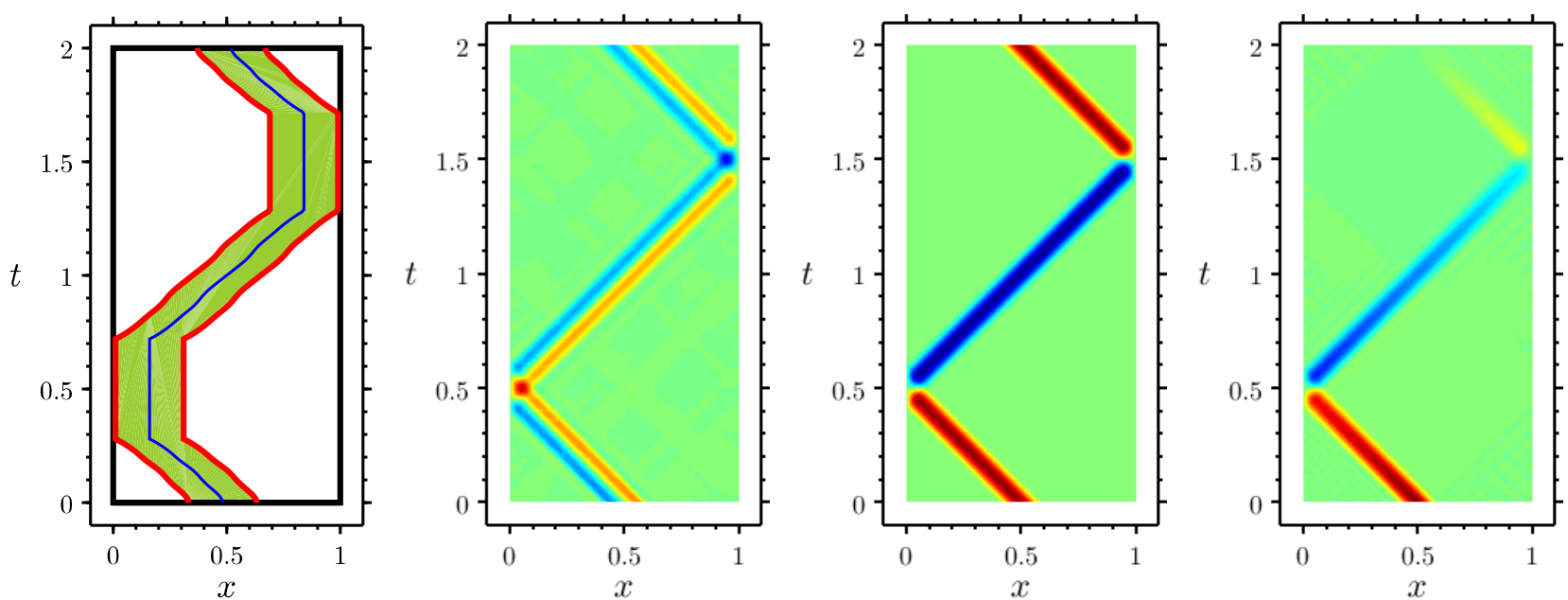

Figure 9. (EX2) - From left to right, optimal control domain, isovalues of the corresponding adjoint state $\varphi$, isovalues of the uncontrolled and controlled wave over the optimal domain, for the initial curve $\gamma_{0} \equiv 1 / 2$.

for cylindrical domains is $\min _{x_{0}} J_{\epsilon}\left(x_{0}\right) \approx 179.22$ leading to a performance index $\Pi\left(\gamma_{\text {opt }}\right) \approx 72.83 \%$. The noncylindrical setup is in that case much more efficient that the cylindrical one. This is due to the fact that the domains we consider can follow very closely the propagation of the travelling wave. This can be noticed in Figure 9, where we display the optimal control domain, the corresponding adjoint state $\varphi$, the uncontrolled and controlled solutions over the optimal domain.

The evolution of the cost $J_{\epsilon}^{n}$ and the derivative $\mathrm{d} J_{\epsilon}^{n}$ with respect to $n$ are displayed in Figure 10. Figure 7Right depicts the values of the functional $J_{\epsilon}$ for the constant curves $\gamma \equiv x_{0}$ used to determine the best cylindrical domain and highlights the low variation of the cost with respect to the position of such domains.

This second example allows to emphasize the sensitivity of the optimal curve with respect to the initial condition. For any $\delta$, consider the initial condition $\left(y_{0}^{\delta}, y_{1}^{\delta}\right)=\left(y_{0}, y_{1}\right)+\delta\left(w_{0}, w_{1}\right)$, where $\left(y_{0}, y_{1}\right)$ is given by (EX2) and supported in the interval [0.4, 0.6], and where $\left(w_{0}, w_{1}\right)$ is any initial condition supported for instance in $[0.8,0.9]$. For $\delta=0$ and any $T>0$, the optimal curve $\gamma_{\text {opt }}$ follows the travelling wave starting in the interval $[0.4,0.6]$. On the other hand, for any $\delta \neq 0$, the optimal curve $\gamma_{\mathrm{opt}}^{\delta}$ will also have to cross the travelling waves generated by the initial condition $\left(w_{0}, w_{1}\right)$ starting in $[0.8,0.9]$. If the controllability time $T$ is small enough, 

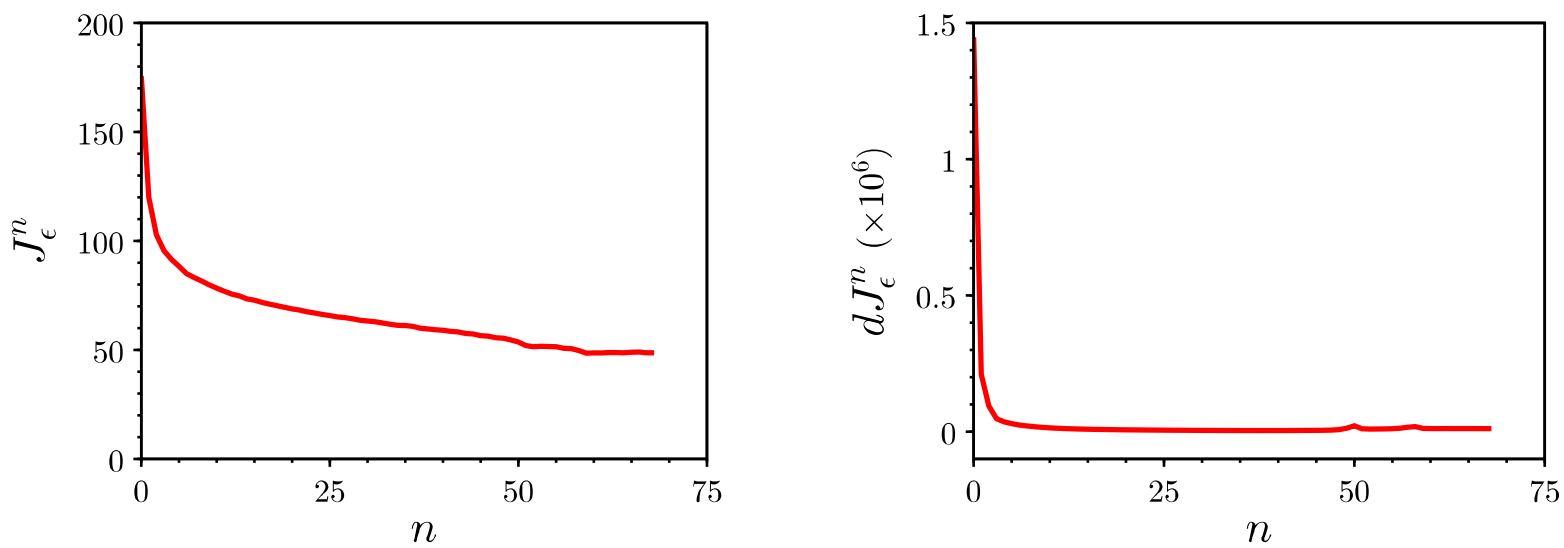

Figure 10. (EX2) - Evolution of the cost $J_{\epsilon}^{n}$ (left) and the derivative d $J_{\epsilon}^{n}$ (right) for the initial curve $\gamma_{0} \equiv 1 / 2$.

TABLE 3. (EX3) - Evolution of the optimal cost $J_{\epsilon}\left(\gamma_{\text {opt }}\right)$ w.r.t. the controllability time $T$.

\begin{tabular}{ccccc}
\hline$T$ & 2 & 1.5 & 1 & 0.5 \\
\hline$J_{\epsilon}\left(\gamma_{\text {opt }}\right)$ & 41.02 & 58.02 & 94.78 & 239 \\
\hline
\end{tabular}

these additional travelling waves remain far from the initial wave. It follows that, for any $\delta \neq 0$, the resulting optimal curve $\gamma_{\mathrm{opt}}^{\delta}$ will be not arbitrarily close to $\gamma_{\mathrm{opt}}$.

- We now consider the initial datum $\left(y_{0}, y_{1}\right)$ given by

$$
y_{0}(x)=(10 x-4)^{2}(10 x-6)^{2} \mathbb{1}_{[0.4,0.6]}(x), \quad y_{1}(x)=0, \quad \text { for } x \in(0,1)
$$

This initial condition generates two waves travelling waves in opposite directions, as can be seen in Figure 11.3. For $T=2, \epsilon=10^{-2}$ and $\rho=10^{-4}$, we initialize the algorithm with the initial curve $\gamma_{0} \equiv 1 / 2$. The convergence is observed after 111 iterations leading to $J_{\epsilon}\left(\gamma_{\text {opt }}\right) \approx 41.02$. Moreover, the minimal cost for cylindrical domains is $\min _{x_{0}} J_{\epsilon}\left(x_{0}\right) \approx 85.08$ leading to a performance index $\Pi\left(\gamma_{\text {opt }}\right) \approx 51.79 \%$. Once again, our non-cylindrical setup is much more efficient than the cylindrical one. It is still due to the fact that the domains we consider can follow the propagation of the travelling waves. This can be noticed in Figure 11, where we display the optimal control domain, the corresponding adjoint state $\varphi$, the uncontrolled and the controlled wave over the optimal domain.

In order to emphasize the influence of the controllability time on the optimal domain, for $\epsilon=10^{-2}$ and $\gamma_{0} \equiv 1 / 2$, we use the descent algorithm with $T=1$ and $\rho=2.5 \times 10^{-5}$, initialized with the curve $\gamma_{0} \equiv 1 / 2$. Remark that the corresponding domain satisfies the geometric optic condition. The convergence is observed after 213 iterations and the optimal cost is $J_{\epsilon}\left(\gamma_{\text {opt }}\right) \approx 94.78$. Moreover, the minimal cost for cylindrical domains is $\min _{x_{0}} J_{\epsilon}\left(x_{0}\right) \approx 183.98$ and the performance index is $\Pi\left(\gamma_{\mathrm{opt}}\right) \approx 48.48 \%$. Observe that the cylindrical domains associated with $x_{0} \notin(0.25,0.75)$ do not verify the geometric optic condition. This highlights the necessity to use non-cylindrical domains. Compared to the simulation for $T=2$, the optimal cost increases by a factor around 2.3. Figure 12 displays the optimal control domain, the corresponding adjoint state $\varphi$, the uncontrolled and controlled wave over the optimal domain. We remark that the projection of the optimal domain on the $x$-axis covers the whole domain $\Omega$, in contrast with the domain associated with $T=2$.

In Table 3, we illustrate the influence of the controllability time $T$ on the optimal cost $J_{\epsilon}\left(\gamma_{\text {opt }}\right)$. More exactly, we remark that the cost is of order $\mathcal{O}\left(T^{-1.27}\right)$. 

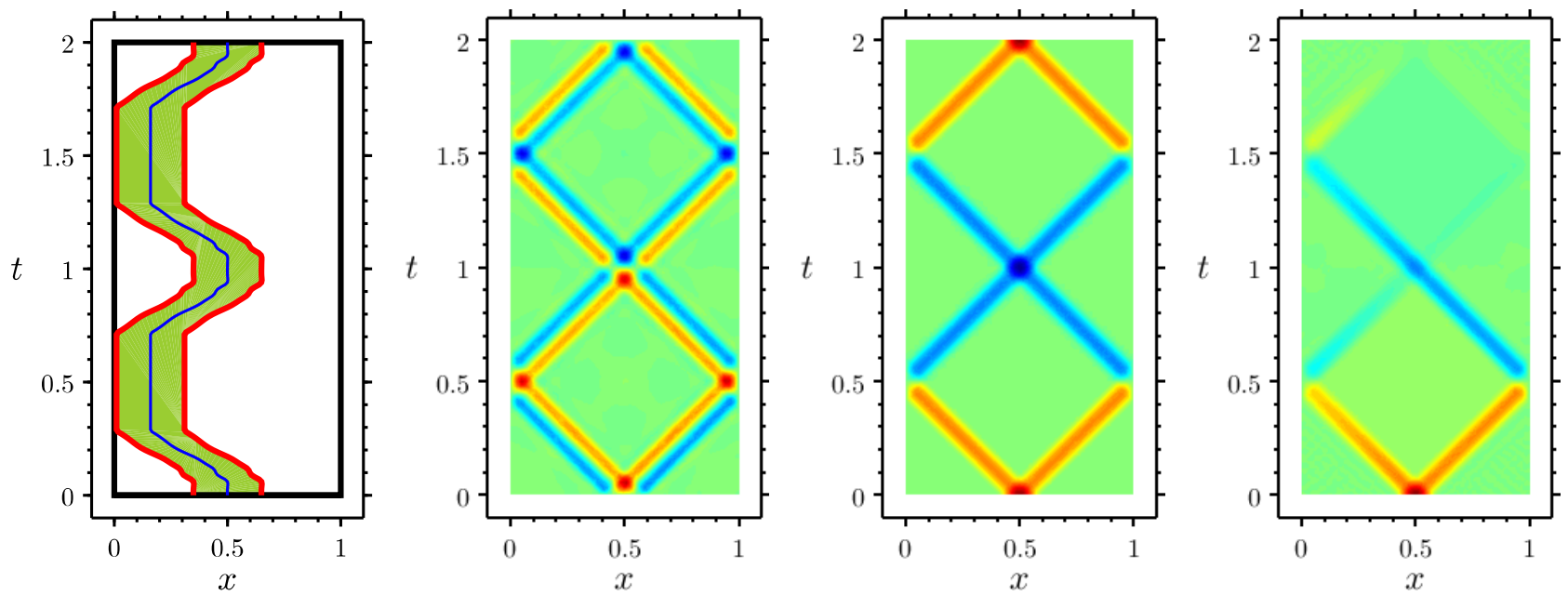

FIGURE 11. (EX3) - From left to right, optimal control domain, isovalues of the corresponding adjoint state $\varphi$, isovalues of the uncontrolled and controlled wave over the optimal domain, for $T=2$, for the initial curve $\gamma_{0} \equiv 1 / 2$.
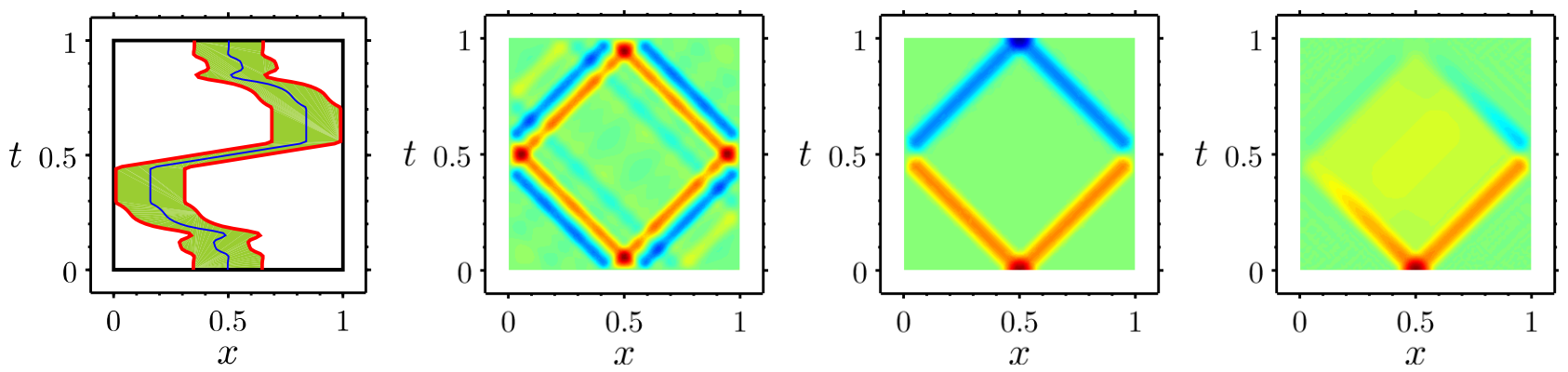

Figure 12. (EX3) - From left to right, optimal control domain, isovalues of the corresponding adjoint state $\varphi$, isovalues of the uncontrolled and controlled wave over the optimal domain, for $T=1$, for the initial curve $\gamma_{0} \equiv 1 / 2$.

- Eventually, in order to highlight the influence of the regularization parameter $\epsilon$ on the optimal domain, we now consider the initial datum $\left(y_{0}, y_{1}\right)$ given by

$$
y_{0}(x)=\left\{\begin{array}{cl}
3 x & \text { if } 0 \leq x \leq 1 / 3 \\
3(1-2 x) & \text { if } 1 / 3 \leq x \leq 2 / 3, \\
-3(1-x) & \text { if } 2 / 3 \leq x \leq 1
\end{array} \quad y_{1}(x)=0, \quad \text { for } x \in(0,1)\right.
$$

For $T=2$ and $\rho=10^{-5}$, we initialize the descent algorithm with the curve $\gamma \equiv 1 / 2$ and consider $\epsilon=10^{-2}$ and $\epsilon=0$. The numbers of iterations until convergence, the values of the functional $J_{\epsilon}$ evaluated at the optimal curve $\gamma_{\text {opt }}$ and the performance indices of $\gamma_{\text {opt }}$ are listed in Table 4. For the initial datum (EX4), the minimal cost for cylindrical domains is $\min _{x_{0}} J_{\epsilon}\left(x_{0}\right) \approx 47.71$.

In Figure 13, we clearly see the regularizing effect of $\epsilon$ and the need of regularization in this case, as the optimal domain obtained when $\epsilon=0$ is very oscillating. Actually, since the initial position $y_{0}$ is supported on the whole domain, the corresponding uncontrolled solution is not localized on a small part of $Q_{T}$ (in contrast for instance to the second example, see (EX2)). The optimal domain is very likely composed of many parts 
TABle 4. (EX4) - Number of iterations, optimal value of the functional $J_{\epsilon}$ and performance index, for $\epsilon \in\left\{0,10^{-2}\right\}$, for the initial curve $\gamma_{0} \equiv 1 / 2$.

\begin{tabular}{ccc}
\hline$\epsilon$ & 0 & $10^{-2}$ \\
\hline Number of iterations & 247 & 389 \\
$J_{\epsilon}\left(\gamma_{\text {opt }}\right)$ & 60.35 & 43.23 \\
$\Pi\left(\gamma_{\text {opt }}\right)$ & $-26.51 \%$ & $9.38 \%$ \\
\hline
\end{tabular}
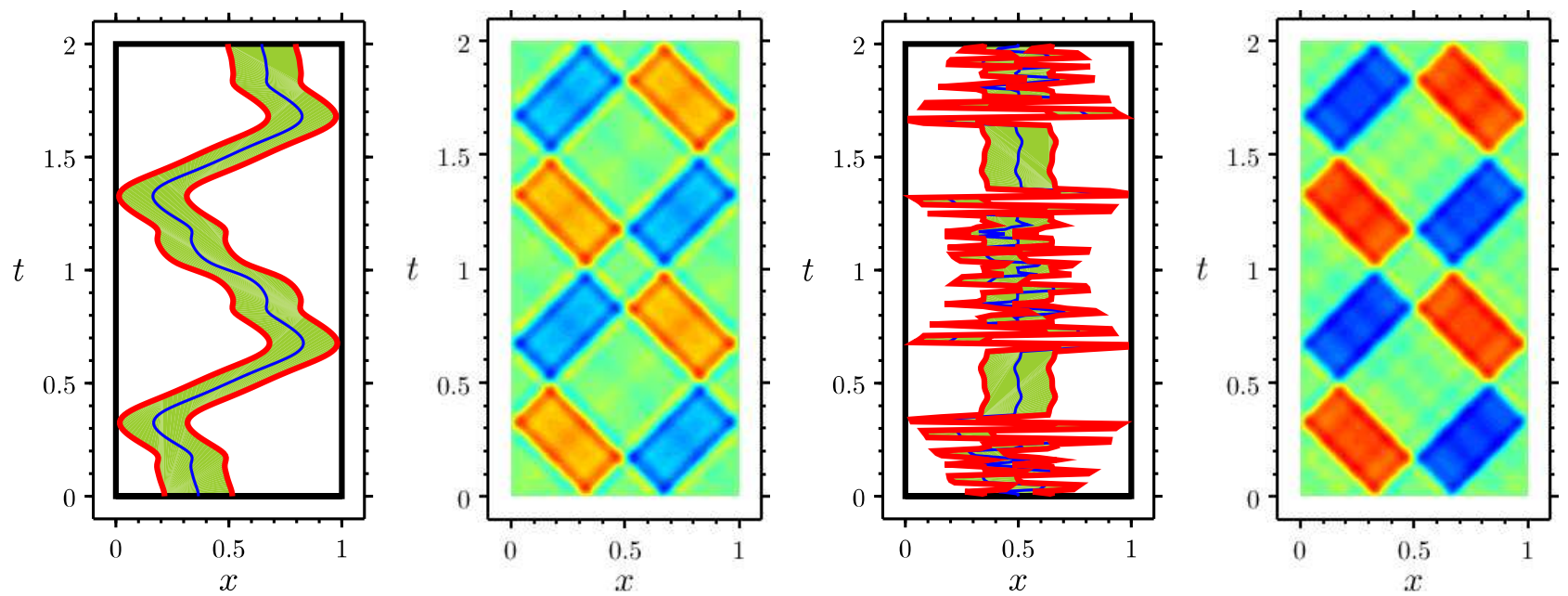

Figure 13. (EX4) - Optimal control domain and isovalues of the adjoint state for $\epsilon=10^{-2}$ (left), $\epsilon=0$ (right), for the initial curve $\gamma_{0} \equiv 1 / 2$.

distributed in $Q_{T}$ leading an homogenization phenomenon. Consequently, when $\epsilon=0$, the algorithm tends to reach these parts leads to large oscillations of the curve $\gamma$. The $\epsilon$-term aims to reduces these oscillation by imposing regularity property of the curves $\gamma$ describing the domains $q_{\gamma}$. In this topic, we refer to the seminal work of Chenais [7]. Let us also mention that a well posedness result is achieved in [29] by introducing a relaxation of the problem 1.4, i.e. by replacing characteristic functions by densities (see also [26] in the context of stabilization of the wave equation).

\subsection{Approximation of the observability constant by an iterative method}

In this last part, we formally describe and use an algorithm allowing to approximate the observability constant appearing in (1.2), associated with any domain $q \subset Q_{T}$. The algorithm is based on the following characterization:

$$
C_{\mathrm{obs}}(q)=\sup _{\mathbf{y}_{0} \in \mathbf{V}} \frac{\left\langle R \Lambda_{q} \mathbf{y}_{0}, \mathbf{y}_{0}\right\rangle_{\mathbf{V}}}{\left\|\mathbf{y}_{0}\right\|_{\mathbf{V}}^{2}}
$$

where $\Lambda_{q}$ and $R$ are respectively the control operator associated with the domain $q$ and the duality operator between the space $\mathbf{W}$ and $\mathbf{V}$ :

$$
\Lambda_{q}:\left\{\begin{array}{rll}
\mathbf{V} & \rightarrow & \mathbf{W} \\
\mathbf{y}_{0} & \mapsto & \widehat{\varphi}_{0}
\end{array}, \quad R:\left\{\begin{array}{ccc}
\mathbf{W} & \rightarrow & \mathbf{V} \\
\left(\varphi_{0}, \varphi_{1}\right) & \mapsto & \left(\left(-\partial_{x}^{2}\right)^{-1} \varphi_{1},-\varphi_{0}\right)
\end{array}\right.\right.
$$





Figure 14. Most expensive initial data $y_{0}$ (left) and $y_{1}$ (right) to be controlled.

In the definition of $\Lambda_{q}, \widehat{\varphi}_{0} \in \mathbf{W}$ is the minimum of the functional $\mathcal{J}^{\star}$ (see (3.4)) associated with $\mathbf{y}_{0} \in \mathbf{V}$. The characterization (4.2) can be obtained by following the steps of Section 2 in [25] and Remark 2.98 in [10]. The main consequence of this characterization is that $C_{\text {obs }}(q)$ can be viewed as the largest eigenvalue of the operator $R \Lambda_{q}$ in $\mathbf{V}$. Consequently, we can formally adapt the power iteration method to our infinite-dimensional setting. The algorithm reads as follows. Let $\mathbf{y}_{0}^{0} \in \mathbf{V}$ be given such that $\left\|\mathbf{y}_{0}^{0}\right\|_{\mathbf{V}}=1$. For $n \geq 0$, using the spacetime finite element method described in Section 3-4 of [6], we compute $\widehat{\boldsymbol{\varphi}}_{0}^{n}=\Lambda_{q} \mathbf{y}_{0}^{n}$ then set $\mathbf{z}_{0}^{n}=R \widehat{\boldsymbol{\varphi}}_{0}^{n}$ and $\mathbf{y}_{0}^{n+1}=\mathbf{z}_{0}^{n} /\left\|\mathbf{z}_{0}^{n}\right\|_{\mathbf{v}}$. We finally have $C_{\text {obs }}(q)=\lim _{n \rightarrow \infty}\left\|\mathbf{z}_{0}^{n}\right\|_{\mathbf{v}}$ while $\mathbf{y}_{0}^{n}$ converges in $\mathbf{V}$ to the most expensive initial datum to control. For the control domain of Figure 3, this algorithm initialized with $\mathbf{y}_{0}^{0}=K(x(1-x), 0)$, $K$ such that $\left\|\mathbf{y}_{0}^{0}\right\|_{\mathbf{V}}=1$, produces the following sequence $\left\{\left\|\mathbf{z}_{0}^{n}\right\|_{\mathbf{V}}\right\}_{n \geq 0}=\{2.689,3.829,3.981,3.994,3.997, \cdots\}$ converging toward the value 4 , in agreement with the result of Section 2.2 based on a graph argument. The most expensive initial datum to be controlled is displayed in Figure 14. Remark that the initial datum solution of (4.2) is not unique.

\section{Conclusion AND PERspeCtives}

Making use of the d'Alembert formula for the solutions of the one dimensional wave equation, we have shown a uniform observability inequality with respect to a class of non cylindrical observation domains satisfying the geometric optic condition. The proof based on arguments from graph theory allows notably to relate the value of the observability constant to the spectrum of the Laplacian matrix, defined in term of the graph associated to any domain $q \subset Q_{T}$. The uniform observability property then allows to consider and analyze the problem of the control's optimal support associated to fixed initial conditions. For simplicity, the optimization is made over connected domains defined by regular curves. As expected, the optimal domains (approximated within a space-time finite element method) are closely related to the travelling waves generated by the initial conditions.

This work may be extended to several directions. First, the characterization of the observability constant in term of a computable eigenvalue problem in Section 4.2 may allow to consider the optimization of such constant with respect to the domain of observation, i.e. $\inf _{q \in \mathcal{Q}_{\text {ad }}^{e}} C_{\text {obs }}(q)$ (as done in [31]). The domain providing the smallest observability constant is nothing else than the trajectory minimizing the cost of control, i.e. the norm of the control corresponding to the worst controllable initial datum. Moreover, from an approximation point of view, we may also consider more general domains (than connected ones) and use, for instance, a level-set method to describe the geometry (as done in [23]). Eventually, this work may be adapted to the case of controls supported on single curves of $Q_{T}$, using the uniform observability property given in [5]. We refer to [3] for a numerical study in this case. The extension of this work to the multi-dimensional case studied in $[17,32]$ is also a challenge. 


\section{REFERENCES}

[1] H. Banks, W. Fang, R. Silcox and R. Smith, Approximation methods for control of structural acoustics models with piezoceramic actuators. J. Intell. Mater. Syst. Struct. 4 (1993) 98-116.

[2] C. Bardos, G. Lebeau and J. Rauch, Sharp sufficient conditions for the observation, control, and stabilization of waves from the boundary. SIAM J. Control Optim. 30 (1992) 1024-1065.

[3] A. Bottois, Pointwise moving control for the 1-D wave equation - Numerical approximation and optimization of the support. To appear in Radon Series on Computational and Applied Mathematics. De Gruyter, In press. https://hal.archives-ouvertes. fr/hal-02972968 (2021).

[4] A.E. Brouwer and W.H. Haemers, Spectra of graphs, Universitext. Springer, New York (2012).

[5] C. Castro, Exact controllability of the 1-D wave equation from a moving interior point. ESAIM: COCV 19 (2013) 301-316.

[6] C. Castro, N. Cîndea and A. Münch, Controllability of the linear one-dimensional wave equation with inner moving forces. SIAM J. Control Optim. 52 (2014) 4027-4056.

[7] D. Chenais, On the existence of a solution in a domain identification problem. J. Math. Anal. Appl. 52 (1975) $189-219$.

[8] F.R.K. Chung, Spectral graph theory. Vol. 92 of CBMS Regional Conference Series in Mathematics. Published for the Conference Board of the Mathematical Sciences, Washington, DC; by the American Mathematical Society, Providence, RI (1997).

[9] N. Cîndea and A. Münch, A mixed formulation for the direct approximation of the control of minimal $L^{2}$-norm for linear type wave equations. Calcolo $\mathbf{5 2}$ (2015) 245-288.

[10] J.-M. Coron, Control and nonlinearity. Vol. 136 of Mathematical Surveys and Monographs. American Mathematical Society, Providence, RI (2007).

[11] L. Cui, X. Liu and H. Gao, Exact controllability for a one-dimensional wave equation in non-cylindrical domains. J. Math. Anal. Appl. 402 (2013) 612-625.

[12] P. Destuynder, I. Legrain, L. Castel and N. Richard, Theoretical, numerical and experimental discussion on the use of piezoelectric devices for control-structure interaction. Eur. J. Mech. A. Solids 11 (1992) 181-213.

[13] B.H. Haak and D.-T. Hoang, Exact observability of a 1-dimensional wave equation on a noncylindrical domain. SIAM J. Control Optim. 57 (2019) 570-589.

[14] F. Hecht, New development in Freefem++. J. Numer. Math. 20 (2012) 251-265.

[15] A. Henrot and M. Pierre, Variation et optimisation de formes. Une analyse géométrique. [A geometric analysis]. Vol. 48 of Mathématiques \& Applications (Berlin) [Mathematics \& Applications]. Springer, Berlin (2005).

[16] A.Y. Khapalov, Controllability of the wave equation with moving point control. Appl. Math. Optim. 31 (1995) $155-175$.

[17] J. Le Rousseau, G. Lebeau, P. Terpolilli and E. Trélat, Geometric control condition for the wave equation with a time-dependent observation domain. Anal. Partial Differ. Equ. 10 (2017) 983-1015.

[18] J.-L. Lions, Contrôlabilité exacte, perturbations et stabilisation de systèmes distribués. Contrôlabilité exacte. [Exact controllability], With appendices by E. Zuazua, C. Bardos, G. Lebeau and J. Rauch. Tome 1. Vol. 8 of Recherches en Mathématiques Appliquées [Research in Applied Mathematics]. Masson, Paris (1988).

[19] K. Liu and J. Yong, Rapid exact controllability of the wave equation by controls distributed on a time-variant subdomain. Chin. Ann. Math. Ser. B 20 (1999) 65-76. A Chinese summary appears in Chin. Ann. Math. Ser. A 20 (1999) 142.

[20] K.A. Lurie, An introduction to the mathematical theory of dynamic materials. Vol. 15 of Advances in Mechanics and Mathematics. Second edition, Springer, Cham (2017) MR2305885.

[21] P. Martin, L. Rosier and P. Rouchon, Null controllability of the structurally damped wave equation with moving control. SIAM J. Control Optim. 51 (2013) 660-684.

[22] B. Mohar, The Laplacian spectrum of graphs. Vol. 2 of Graph theory, combinatorics, and applications. (Kalamazoo, MI, 1988). Wiley-Intersci. Publ., Wiley, New York (1991) 871-898.

[23] A. Münch, Optimal design of the support of the control for the 2-D wave equation: a numerical method. Int. J. Numer. Anal. Model. 5 (2008) 331-351.

[24] A. Münch, Optimal location of the support of the control for the 1-D wave equation: numerical investigations. Comput. Optim. Appl. 42 (2009) 443-470.

[25] A. Münch, Numerical estimations of the cost of boundary controls for the equation $y_{t}-\varepsilon y_{x x}+M y_{x}=0$ with respect to $\varepsilon$, in Recent advances in PDEs: analysis, numerics and control. Vol. 17 of SEMA SIMAI Springer Ser. Springer, Cham (2018) 159-191.

[26] A. Münch, P. Pedregal and F. Periago, Optimal design of the damping set for the stabilization of the wave equation. J. Differ. Equ. 231 (2006) 331-358.

[27] A.O. Özer, Potential formulation for charge or current-controlled piezoelectric smart composites and stabilization results: electrostatic versus quasi-static versus fully-dynamic approaches. IEEE Trans. Automat. Control 64 (2019) 989-1002.

[28] A.O. Özer and K.A. Morris, Modeling and stabilization of current-controlled piezo-electric beams with dynamic electromagnetic field. ESAIM:COCV 26 (2020) 24.

[29] F. Periago, Optimal shape and position of the support for the internal exact control of a string. Systems Control Lett. 58 (2009) 136-140.

[30] Y. Privat, E. Trélat and E. Zuazua, Optimal location of controllers for the one-dimensional wave equation. Ann. Inst. Henri Poincaré Anal. Non Linéaire 30 (2013) 1097-1126. 
[31] Y. Privat, E. Trélat and E. Zuazua, Optimal observation of the one-dimensional wave equation. J. Fourier Anal. Appl. 19 (2013) 514-544.

[32] A. Shao, On Carleman and observability estimates for wave equations on time-dependent domains. Proc. Lond. Math. Soc. 119 (2019) 998-1064.

[33] M. Tucsnak, Control of plate vibrations by means of piezoelectric actuators. Discrete Contin. Dynam. Syst. 2 (1996) $281-293$. 